\title{
Reconstruction of an unknown inclusion by thermography
}

\author{
Victor IsAKov, KyOUNGSUn KIM AND GEN NAKAMURA
}

\begin{abstract}
We establish a probe type reconstruction scheme for identifying an inclusion inside a heat conductive medium by nondestructive testing called thermography. For the one space dimension, this has been already achieved by Y. Daido, H. Kang and G. Nakamura. The present paper shows that their result can be generalized to higher space dimension.
\end{abstract}

Mathematics Subject Classification (2010): 35R30.

\section{Introduction}

Thermography is a nondestructive testing to extract the information of unknown cracks, cavities and inclusions inside a heat conductor. This is a typical inverse problem in industrial and medical engineering. The information we want to know via thermography are their size, location etc. The procedure of thermography is to apply a heat flux (sometimes called thermal load) to the surface of the heat conductor and measure the resulting temperature over certain time. This procedure can be repeated several times and we can superpose the measured data. For more details, we refer $[3,16,17]$.

In this paper, we are concerned with a mathematical study of thermography. In particular, we want to recover an unknown inclusion inside a heat conductor by thermography. Under some mathematically idealized situation, we will provide a mathematically rigorous scheme to reconstruct the unknown inclusion by boundary measurements. In the rest of this introduction, we first introduce several notations used throughout this paper to formulate our inverse problem and refer to the known results on the problem.

Let $\Omega$ be a bounded domain in $\mathbb{R}^{n}(1 \leq n \leq 3)$ with $C^{2}$ boundary if $n \geq 2$. We consider a heat conductor $\Omega$ with an inclusion $D$ such that $\bar{D} \subset \Omega, \Omega \bar{\backslash} \bar{D}$ is connected and $\partial D$ is of class $C^{1, \alpha}(0<\alpha<1)$ for simplicity. Let the heat

The first author was in part supported by the NSF grant DMS 07-07734 and Emylou Keith and Betty Dutcher Distinguished Professorship at the Wichita State University. The second author was supported by the Korea Research Foundation Grant funded by the Korean Government (MOEHRD) (KRF-2006-214-C00007). The third author was partially supported by Grant-in-Aid for Scientific Research (B)(No. 19340028) of Japan Society for Promotion of Science.

Received March 6, 2008; accepted in revised form November 13, 2009. 
conductivity $\gamma(x)$ in $\Omega$ be given as follows:

$$
\gamma(x)= \begin{cases}1 & \text { for } x \in \Omega \backslash \bar{D}, \\ k & \text { for } x \in D,\end{cases}
$$

where $k$ is a positive constant which is not 1 . That is, by using the characteristic function $\chi_{D}$ of $D, \gamma(x)$ is given as $\gamma(x)=1+(k-1) \chi_{D}$. This is the most simplest case for the heat conductivity. We will comment later at the end of this section on more general case for the heat conductivity.

For simplicity, we denote $B \times\left(T_{1}, T_{2}\right)$ and $B \times(0, T)$ by $B_{\left(T_{1}, T_{2}\right)}$ and $B_{T}$, respectively, for a set $B . H^{q}(\Omega), H^{p}(\partial \Omega)$ and $H^{q, r}\left(\Omega_{T}\right)$ with $q, r \in \mathbb{Z}_{+}:=\mathbb{N} \cup\{0\}$ and $p=\frac{1}{2}$ denote the usual Sobolev spaces, where $q$ and $r$ in $H^{q, r}\left(\Omega_{T}\right)$ denote the regularity with respect to $x$ and $t$, respectively. Also, for an open set $U \subset \mathbb{R}^{n+1}$, $q, r \in \mathbb{Z}_{+}, H^{q, r}(U)$ is defined likewise $H^{q, r}\left(\Omega_{T}\right)$. That is, $g \in H^{q, r}(U)$ if the norm

$$
\|g\|_{H^{q, r}(U)}:=\left(\sum_{\substack{|\alpha|+2 k \leq q \\ k \leq r}} \int_{U}\left|\partial_{x}^{\alpha} \partial_{t}^{k} g\right|^{2} \mathrm{~d} t \mathrm{~d} x\right)^{1 / 2}
$$

is finite. A function $f(x, t)$ is said to be in $L^{2}((0, T) ; X)$ if $f(\cdot, t)$ takes a value in a Hilbert space $X$ for almost all $t \in(0, T)$ and

$$
\|f\|_{L^{2}((0, T) ; X)}^{2}=\int_{0}^{T}\|f(\cdot, t)\|_{X}^{2} \mathrm{~d} t<\infty .
$$

Let $W\left(\Omega_{T}\right):=\left\{v \in L^{2}\left((0, T) ; H^{1}(\Omega)\right) ; \partial_{t} v \in L^{2}\left((0, T) ;\left(H^{1}(\Omega)\right)^{*}\right)\right\}$, where $\left(H^{1}(\Omega)\right)^{*}$ is the dual space of $H^{1}(\Omega)$.

In this paper, we also use the Hörmander's notation for function spaces to handle the mixed type boundary condition. Namely, when $X$ is an ambient space, let $Y$ be a subspace of $X$ where $\partial Y \neq \varnothing$ and $\partial Y$ is of Lipschitz class. Then a space $\bar{H}^{p}(Y)$ consists of distributions which have extension to $X$ and we shall write $H^{p}(\bar{Y})$ for a space of distributions in $H^{p}(X)$ supported by $\bar{Y}$. It is well known that there are dualities. For dual spaces $\left(\bar{H}^{p}(Y)\right)^{*},\left(\dot{H}^{p}(\bar{Y})\right)^{*}$ of $\bar{H}^{p}(Y), \dot{H}^{p}(\bar{Y})$, we use

$$
\dot{H}^{-p}(\bar{Y}):=\left(\bar{H}^{p}(Y)\right)^{*}, \quad \bar{H}^{-p}(Y):=\left(\dot{H}^{p}(\bar{Y})\right)^{*} .
$$

(See Hörmander's book [10].)

Let $\partial \Omega$ consist of two parts. Namely,

$$
\partial \Omega=\overline{\Gamma^{D}} \cup \overline{\Gamma^{N}}
$$

where $\Gamma^{D}$ and $\Gamma^{N}$ are open subsets of $\partial \Omega$ such that $\Gamma^{D} \cap \Gamma^{N}=\emptyset$ and for $n=3$, the boundaries $\partial \Gamma^{D}$ of $\Gamma^{D}$ and $\partial \Gamma^{N}$ of $\Gamma^{N}$ are $C^{2}$ if they are nonempty. 
Now consider a boundary value problem to find a unique weak solution $u=$ $u(f, g) \in L^{2}\left((0, T) ; H^{1}(\Omega)\right)$ which satisfies

$$
\left\{\begin{array}{l}
\mathcal{P}_{D} u(x, t):=\partial_{t} u(x, t)-\operatorname{div}_{x}\left(\gamma(x) \nabla_{x} u(x, t)\right)=0 \quad \text { in } \Omega_{T} \\
u(x, t)=f(x, t) \quad \text { on } \Gamma_{T}^{D}, \quad \partial_{\nu} u(x, t)=g(x, t) \quad \text { on } \Gamma_{T}^{N}, \\
u(x, 0)=0 \quad \text { for } x \in \Omega
\end{array}\right.
$$

for a given $f \in L^{2}\left((0, T) ; \bar{H}^{\frac{1}{2}}\left(\Gamma^{D}\right)\right)$ and $g \in L^{2}\left((0, T) ; \dot{H}^{-\frac{1}{2}}\left(\overline{\Gamma^{N}}\right)\right)$. In another words, by assuming the initial temperature of a heat conductive medium $\Omega$ is 0 and the temperature on $\Gamma_{T}^{D}$ is $f$, it is to determine the temperature $u=u(f, g)$ induced in $\Omega_{T}$ after applying the heat flux $g$ on $\Gamma_{T}^{N}$. Here $\partial_{\nu} u=\nabla u \cdot v$ with outward unit normal vector $v$ to $\partial \Omega$.

It is well known that the boundary value problem (1.2) is well posed( [19]). That is, there exists a unique solution $u=u(f, g) \in L^{2}\left((0, T) ; H^{1}(\Omega)\right)$ to (1.2). Further, if $\Gamma^{D} \neq \emptyset$, and $f(\cdot, t)=0=g(\cdot, t)\left(t>T^{\prime}\right)$ with $0<T^{\prime}<T$, then $u(f, g)$ has the decaying property. That is $u(f, g)$ decays exponentially after $t=T^{\prime}$. Based on the fact that (1.2) is well posed, we define the Neumann-toDirichlet map $\Lambda_{D}$ as follows. For fixed $f \in L^{2}\left((0, T) ; \bar{H}^{\frac{1}{2}}\left(\Gamma^{D}\right)\right)$, define

$$
\begin{aligned}
\Lambda_{D}: L^{2}\left((0, T) ; \dot{H}^{-\frac{1}{2}}\left(\overline{\Gamma^{N}}\right)\right) & \rightarrow L^{2}\left((0, T) ; \bar{H}^{\frac{1}{2}}\left(\Gamma^{N}\right)\right) \\
g & \left.\mapsto u(f, g)\right|_{\Gamma_{T}^{N}} .
\end{aligned}
$$

Now, we take the Neumann-to-Dirichlet map $\Lambda_{D}$ as measured data. Then, our inverse problem is to reconstruct the unknown inclusion $D$ from $\Lambda_{D}$. This means that by fixing the temperature on $\Gamma^{D}$ to $f$, we repeat conducting a measurement many times which puts a heat flux $g$ on $\Gamma^{N}$ and measure the corresponding temperature on $\Gamma^{N}$ over the time interval $(0, T)$. Taking the map $\Lambda_{D}$ as measured data is of course impractical. However, when $\Gamma^{D} \neq \emptyset$ and $f=0$, we can actually repeat the aforementioned measurement many times due to the decaying property of $u(f, g)$. We can even superpose the measured data to generate $\Lambda_{D}$ approximately.

The uniqueness and stability of identifying $D$ from $\Lambda_{D}$ has been already proved in [7] and [6], respectively. Further, as for the reconstruction Y. Daido, H. Kang, and G. Nakamura [4] gave a reconstruction scheme for one space dimensional case. It is an analogue of the probe method which was introduced by M. Ikehata [11] to identify the shape of unknown inclusion in a stationary heat conductive medium. They developed a theory how to adapt the probe method for the stationary heat conductive case in one space dimension to the dynamical heat conductive case in one space dimension. We will call their theory dynamical probe method.

The main purpose of this paper is to generalize the result of [4] to higher dimension in the case that the inclusion $D$ does not depend on $t$. The new ingredients of this paper are the followings:

(i) We slightly modified the definition of the indicator function introduced in [4] for our purpose. Here, the indicator function is a mathematical testing machine to identify $D$ and it is defined in terms of the measured data $\Lambda_{D}$. 
(ii) We gave an explicit form for the dominant part of the reflected solution, which is the key to analyze the behavior of the indicator function.

More precisely, indicator function is defined in (2.14) as a limit of a pre-indicator function which is defined in Definition 2.3. By finding a special function called reflected solution (you can find it after Lemma 2.4), we can see that it is enough to know the behavior of the reflected solution to know that of indicator function (Theorem 2.8). For the reflected solution $w_{(y, s)}$, we decompose it in terms of some fundamental solutions as follows:

$$
\begin{aligned}
w_{(y, s)}(x, t)= & \left\{E(x, t ; y, s)-\Gamma_{-}(\Phi(x), t ; \Phi(y), s)\right\}+\left\{\Gamma_{-}(\Phi(x), t ; \Phi(y), s)\right. \\
& -\Gamma(\Phi(x), t ; \Phi(y), s)\}+\{\Gamma(\Phi(x), t ; \Phi(y), s)-\Gamma(x, t ; y, s)\} \\
& +\left\{\Gamma(x, t ; y, s)-V_{(y, s)}(x, t)\right\} .
\end{aligned}
$$

Meanings of each function in this decomposition are explained in Section 3. Specially, the second brace is the dominant part of the reflected solution, which is analyzed in Lemma 3.4.

We would like to remark here that it is possible to develop the dynamical probe method for the heat conductivities of general forms. They can be a piecewise $C^{\mu}(0<\mu<1)$ smooth anisotropic conductivity with discontinuity everywhere across $\partial D$. These further development will be published elsewhere.

The rest of this paper is organized as follows. In Section 2, we will state our main result. Its proof is given in Section 3. appendix includes some detailed computations we postponed in Section 3.

ACKNOWLEDGEMENTS. We would like to thank the referee for many useful comments to improve our paper.

\section{Main result}

For $(y, s),\left(y, s^{\prime}\right) \in \mathbb{R}^{3} \times \mathbb{R}$, let $\Gamma(x, t ; y, s)$ and $\Gamma^{*}\left(x, t ; y, s^{\prime}\right)$ for $(x, t) \in \Omega_{T}$ be

$$
\begin{aligned}
\Gamma(x, t ; y, s) & = \begin{cases}\frac{1}{[4 \pi(t-s)]^{n / 2}} \exp \left[-\frac{|x-y|^{2}}{4(t-s)}\right], & t>s, \\
0, \quad t \leq s,\end{cases} \\
\Gamma^{*}\left(x, t ; y, s^{\prime}\right) & = \begin{cases}0, & t \geq s^{\prime}, \\
\frac{1}{\left[4 \pi\left(s^{\prime}-t\right)\right]^{n / 2}} \exp \left[-\frac{|x-y|^{2}}{4\left(s^{\prime}-t\right)}\right], & t<s^{\prime} .\end{cases}
\end{aligned}
$$

Then, $\mathcal{P}_{\emptyset} \Gamma(x, t ; y, s):=\left(\partial_{t}-\triangle_{x}\right) \Gamma(x, t ; y, s)=0$ if $(x, t) \neq(y, s)$ and $\mathcal{P}_{\emptyset}^{*} \Gamma^{*}\left(x, t ; y, s^{\prime}\right):=\left(-\partial_{t}-\triangle_{x}\right) \Gamma^{*}\left(x, t ; y, s^{\prime}\right)=0$ if $(x, t) \neq\left(y, s^{\prime}\right)$. Let 
$G(x, t ; y, s)$ be a solution of

$$
\left\{\begin{array}{l}
\mathcal{P}_{\emptyset} G(x, t ; y, s)=\delta(x-y) \delta(t-s) \quad \text { in } \quad \Omega_{T}, \\
G(\cdot, \cdot ; y, s)=0 \text { on } \Gamma_{T}^{D}, \\
G(x, t ; y, s)=0 \text { for } x \in \Omega, \quad t \leq s
\end{array}\right.
$$

and $G^{*}\left(x, t ; y, s^{\prime}\right)$ a solution of

$$
\left\{\begin{array}{l}
\mathcal{P}_{\emptyset}^{*} G^{*}\left(x, t ; y, s^{\prime}\right)=\delta(x-y) \delta\left(t-s^{\prime}\right) \quad \text { in } \quad \Omega_{T}, \\
G^{*}\left(\cdot, \cdot ; y, s^{\prime}\right)=0 \text { on } \Gamma_{T}^{D}, \\
G^{*}\left(x, t ; y, s^{\prime}\right)=0 \quad \text { for } \quad x \in \Omega, \quad t \geq s^{\prime}
\end{array}\right.
$$

such that

$$
G(x, t ; y, s)-\Gamma(x, t ; y, s), G^{*}\left(x, t ; y, s^{\prime}\right)-\Gamma^{*}\left(x, t ; y, s^{\prime}\right) \in C^{\infty}\left(\overline{\Omega_{T}}\right) .
$$

Now we prove Runge's approximation theorem. We follow the lines in [4]. But we need a slight modification because we are dealing with mixed type boundary condition.

Theorem 2.1. For $T_{0}^{\prime}<T_{0}<T_{1}<T_{1}^{\prime}$, let $U$ be an open subset of $\Omega_{\left(T_{0}^{\prime}, T_{1}^{\prime}\right)}$ such that

$$
\left\{\begin{array}{c}
\partial U \quad \text { is Lipschitz; } \\
\bar{U} \subset \Omega_{\left(T_{0}^{\prime}, T_{1}^{\prime}\right)} \text { and }\left(\Omega_{\left(T_{0}^{\prime}, T_{1}^{\prime}\right)} \backslash \bar{U}\right) \cap\{t=\theta\} \\
\quad \text { are connected for all } \theta \in\left(T_{0}^{\prime}, T_{1}^{\prime}\right) .
\end{array}\right.
$$

For further reference, we say $U$ satisfies property $(R)$ in $\Omega_{\left(T_{0}^{\prime}, T_{1}^{\prime}\right)}$ if $U$ satisfies (2.3). Then, for any open subset $V$ of $\Omega_{\left(T_{0}^{\prime}, T_{1}^{\prime}\right)}$ such that $\bar{U} \subset V \subset \bar{V} \subset \Omega_{\left(T_{0}^{\prime}, T_{1}^{\prime}\right)}$ and any $v \in H^{2,1}(V)$ satisfying

$$
\left\{\begin{array}{l}
\mathcal{P}_{\emptyset} v=0 \quad \text { in } \quad V \\
v(x, t)=0 \quad \text { for all }(x, t) \in V \quad \text { with } \quad T_{0}^{\prime}<t \leq T_{0}
\end{array}\right.
$$

there exists a sequence $\left\{v^{j}\right\} \subset H^{2,1}\left(\Omega_{\left(T_{0}^{\prime}, T_{1}^{\prime}\right)}\right)$ such that

$$
\left\{\begin{array}{l}
\mathcal{P}_{\emptyset} v^{j}=0 \quad \text { in } \quad \Omega_{\left(T_{0}^{\prime}, T_{1}^{\prime}\right),} \\
v^{j}=0 \text { on } \Gamma^{D} \times\left(T_{0}^{\prime}, T_{1}^{\prime}\right), \\
v^{j}(x, t)=0 \text { for all } \quad(x, t) \in \Omega \times\left(T_{0}^{\prime}, T_{0}\right],
\end{array}\right.
$$

and $v^{j}$ converges to $v$ in $L^{2}(U)$ as $j \rightarrow \infty$. 
Proof. Let $X$ be the collection of all $v \in H^{2,1}(V)$ satisfying (2.4), and $Y$ be the collection of all $u \in H^{2,1}\left(\Omega_{\left(T_{0}^{\prime}, T_{1}^{\prime}\right)}\right)$ satisfying (2.5). We prove that $Y$ is dense in $X$ in $L^{2}(U)$ norm, in other words, that if $f \in L^{2}(U)$ satisfies $(f, u)_{L^{2}(U)}=0$ for all $u \in Y$, then $(f, v)_{L^{2}(U)}=0$ for all $v \in X$.

Suppose that $f \in L^{2}(U)$ satisfies $(f, u)_{L^{2}(U)}=0$ for all $u \in Y$. Define $F$ by

$$
F:= \begin{cases}f & \text { in } U, \\ 0 & \text { in } \Omega_{\left(T_{0}^{\prime}, T_{1}^{\prime}\right)} \backslash \bar{U} .\end{cases}
$$

Then there exists a unique $w \in H^{2,1}\left(\Omega_{\left(T_{0}^{\prime}, T_{1}^{\prime}\right)}\right)$ satisfying

$$
\begin{cases}\mathcal{P}_{\emptyset}^{*} w=F & \text { in } \quad \Omega_{\left(T_{0}^{\prime}, T_{1}^{\prime}\right)} \\ w=0 & \text { on } \quad \partial \Omega_{\left(T_{0}^{\prime}, T_{1}^{\prime}\right)} \\ w\left(x, T_{1}^{\prime}\right)=0 & \text { for } \quad x \in \Omega .\end{cases}
$$

(For a solvability of (2.6), see, for example, [19].) For any $u \in Y$, we have

$$
\begin{aligned}
& 0=\int_{U} u f=\int_{\Omega_{\left(T_{0}^{\prime}, T_{1}^{\prime}\right)}} u \mathcal{P}_{\emptyset}^{*} w \quad \mathrm{~d} x \mathrm{~d} t=\int_{\Omega_{\left(T_{0}^{\prime}, T_{1}^{\prime}\right)}}\left(u \mathcal{P}_{\emptyset}^{*} w-w \mathcal{P}_{\emptyset} u\right) \quad \mathrm{d} x \mathrm{~d} t \\
& =\int_{\partial \Omega_{\left(T_{0}^{\prime}, T_{1}^{\prime}\right)}}\left(w \partial_{\nu} u-u \partial_{\nu} w\right) \quad \mathrm{d} \sigma \mathrm{d} t=-\int_{\partial \Omega_{\left(T_{0}^{\prime}, T_{1}^{\prime}\right)}} u \partial_{\nu} w \quad \mathrm{~d} \sigma \mathrm{d} t .
\end{aligned}
$$

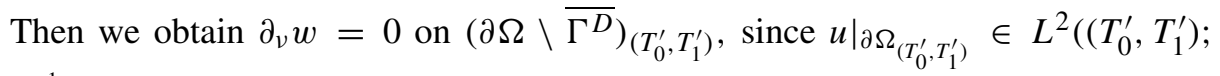
$\left.H^{\frac{1}{2}}(\partial \Omega)\right)$ can be taken arbitrarily except the condition $u=0$ on $\Gamma^{D} \times\left(T_{0}^{\prime}, T_{1}^{\prime}\right)$. Then using the unique continuation property (cf. [12]), we get

$$
w=0 \quad \text { on } \quad \overline{\Omega_{\left(T_{0}^{\prime}, T_{1}^{\prime}\right)} \backslash \bar{U}}
$$

Let $v \in X$. Then, by (2.7), we have

$$
\begin{aligned}
(v, f)_{L^{2}(U)} & =\int_{U} v f=\int_{U}\left(v \mathcal{P}_{\emptyset}^{*} w-w \mathcal{P}_{\emptyset} v\right) \mathrm{d} x \mathrm{~d} t \\
& =\int_{\partial U}\left(w \partial_{\nu} v-v \partial_{\nu} w\right)=0 .
\end{aligned}
$$

Here, we extended the meaning of $\partial_{\nu}$ to denote the outer normal derivative on $\partial U$. This completes the proof. 
Remark 2.2. An analogous theorem can be obtained for the dual problem corresponding to the operator $\mathcal{P}_{\emptyset}^{*}$. By the interior regularity [14, Theorem 7.13], we also have the convergence $v^{j} \rightarrow v$ in $H^{2,1}(U)$ if we shrink $U$ a little bit.

Let $f \in L^{2}\left((0, T) ; \bar{H}^{\frac{1}{2}}\left(\Gamma^{D}\right)\right)$ and $g \in L^{2}\left((0, T) ; \dot{H}^{-\frac{1}{2}}\left(\overline{\Gamma^{N}}\right)\right)$. For arbitrarily given $\varepsilon>0$, let us consider two points $(y, s)$ and $\left(y, s^{\prime}\right)$ in $\Omega_{(-\varepsilon, T+\varepsilon)}$ with $s \neq s^{\prime}$. Then by Theorem 2.1 and Remark 2.2, we can select two sequences of functions $\left\{v_{(y, s)}^{j 0}\right\}$ and $\left\{\psi_{\left(y, s^{\prime}\right)}^{j 0}\right\}$ in $H^{2,1}\left(\Omega_{(-\varepsilon, T+\varepsilon)}\right)$ such that

$$
\begin{cases}\mathcal{P}_{\emptyset} v_{(y, s)}^{j 0}=0 & \text { in } \Omega_{(-\varepsilon, T+\varepsilon)}, \\ v_{(y, s)}^{j 0}=0 & \text { on } \Gamma^{D} \times(-\varepsilon, T+\varepsilon), \\ v_{(y, s)}^{j 0}(x, t)=0 & \text { if }-\varepsilon<t \leq 0, \\ v_{(y, s)}^{j 0} \rightarrow G(\cdot, \cdot ; y, s) & \text { in } \quad H^{2,1}(U) \text { as } \quad j \rightarrow \infty\end{cases}
$$

and

$$
\begin{cases}\mathcal{P}_{\emptyset}^{*} \psi_{\left(y, s^{\prime}\right)}^{j 0}=0 & \text { in } \Omega_{(-\varepsilon, T+\varepsilon)}, \\ \psi_{\left(y, s^{\prime}\right)}^{j 0}=0 & \text { on } \Gamma^{D} \times(-\varepsilon, T+\varepsilon), \\ \psi_{\left(y, s^{\prime}\right)}^{j 0}(x, t)=0 & \text { if } T \leq t<T+\varepsilon, \\ \psi_{\left(y, s^{\prime}\right)}^{j j} \rightarrow G^{*}\left(\cdot, \cdot ; y, s^{\prime}\right) & \text { in } H^{2,1}(U) \text { as } j \rightarrow \infty\end{cases}
$$

for each open set $U$ in $\Omega_{(-\varepsilon, T+\varepsilon)}$ which satisfies property (R) with $T_{0}^{\prime}=-\varepsilon, T_{1}^{\prime}=$ $T+\varepsilon$ and does not contain $(y, s)$ and $\left(y, s^{\prime}\right)$.

Let $v$ and $\psi$ be functions such that

$$
\left\{\begin{array} { l } 
{ \mathcal { P } _ { \emptyset } v = 0 \text { in } \Omega _ { ( - \varepsilon , T + \varepsilon ) , } } \\
{ v = f \text { on } \Gamma ^ { D } \times ( - \varepsilon , T + \varepsilon ) , } \\
{ \partial _ { \nu } v = 0 \text { on } \Gamma ^ { N } \times ( - \varepsilon , T + \varepsilon ) , } \\
{ v ( x , 0 ) = 0 \text { for } x \in \Omega , }
\end{array} \quad \left\{\begin{array}{l}
\mathcal{P}_{\emptyset}^{*} \psi=0 \text { in } \Omega_{(-\varepsilon, T+\varepsilon)}, \\
\psi=0 \text { on } \Gamma^{D} \times(-\varepsilon, T+\varepsilon), \\
\partial_{\nu} \psi=g \text { on } \Gamma^{N} \times(-\varepsilon, T+\varepsilon), \\
\psi(x, T)=0 \text { for } x \in \Omega
\end{array}\right.\right.
$$

For $j=1,2, \cdots$, we define

$$
\left\{\begin{array}{l}
v_{(y, s)}^{j}:=v+v_{(y, s)}^{j 0} \\
\psi_{\left(y, s^{\prime}\right)}^{j}:=\psi+\psi_{\left(y, s^{\prime}\right)}^{j 0}
\end{array} .\right.
$$

Then sequences $\left\{v_{(y, s)}^{j}\right\},\left\{\psi_{\left(y, s^{\prime}\right)}^{j}\right\}$ are to be sequences of approximate functions satisfying

$$
\left\{\begin{array}{l}
v_{(y, s)}^{j} \rightarrow V_{(y, s)}:=v+G(\cdot, \cdot ; y, s) \\
\psi_{\left(y, s^{\prime}\right)}^{j} \rightarrow \Psi_{\left(y, s^{\prime}\right)}:=\psi+G^{*}\left(\cdot, \cdot ; y, s^{\prime}\right)
\end{array}\right.
$$

in $H^{2,1}(U)$ as $j \rightarrow \infty$. 
Definition 2.3. Let $(y, s),\left(y, s^{\prime}\right) \in \Omega_{T}$ be such that $s \neq s^{\prime}$, and $\left\{v_{(y, s)}^{j}\right\},\left\{\psi_{\left(y, s^{\prime}\right)}^{j}\right\} \subset$ $H^{2,1}\left(\Omega_{(-\varepsilon, T+\varepsilon)}\right)$ be sequences of approximate functions given in (2.8). Then, we define the pre-indicator function $I\left(y, s^{\prime} ; y, s\right)$ as follows.

$$
I\left(y, s^{\prime} ; y, s\right)=\left.\lim _{j \rightarrow \infty} \int_{\Gamma_{T}^{N}}\left(\Lambda_{\emptyset}-\Lambda_{D}\right)\left(\left.\partial_{\nu} v_{(y, s)}^{j}\right|_{\Gamma_{T}^{N}}\right) \partial_{\nu} \psi_{\left(y, s^{\prime}\right)}^{j}\right|_{\Gamma_{T}^{N}} \mathrm{~d} \sigma_{x} \mathrm{~d} t
$$

whenever the limit exists.

This pre-indicator function will be used later to define an indicator function which is a mathematical testing machine to identify the unknown inclusion.

For approximate functions $\left\{v_{(y, s)}^{j}\right\} \subset H^{2,1}\left(\Omega_{(-\varepsilon, T+\varepsilon)}\right)$ given in definition above, let $u_{(y, s)}^{j}:=u\left(f,\left.\partial_{v} v_{(y, s)}^{j}\right|_{\left.\Gamma_{T}^{N}\right)}\right.$ and $w_{(y, s)}^{j}:=u_{(y, s)}^{j}-v_{(y, s)}^{j}$. Then, $w_{(y, s)}^{j}$ satisfies the following mixed boundary value problem

$$
\begin{cases}\mathcal{P}_{D} w_{(y, s)}^{j}=(k-1) \operatorname{div}\left(\chi_{D} \nabla_{x} v_{(y, s)}^{j}\right) & \text { in } \Omega_{T}, \\ w_{(y, s)}^{j}=0 \quad \text { on } \Gamma_{T}^{D}, \quad \partial_{\nu} w_{(y, s)}^{j}=0 & \text { on } \Gamma_{T}^{N}, \\ w_{(y, s)}^{j,}(x, 0)=0 \quad \text { for } x \in \Omega . & \end{cases}
$$

The derivation of Lemma 3.2 in [4] gives the following result.

Lemma $2.4([4])$. Let $(y, s) \in(\Omega \backslash \bar{D})_{T}$. Then the sequence $\left\{w_{(y, s)}^{j}\right\}$ has a limit $w_{(y, s)}$ in $W\left(\Omega_{T}\right)$ satisfying

$$
\begin{cases}\mathcal{P}_{D} w_{(y, s)}=(k-1) \operatorname{div}\left(\chi_{D} \nabla V_{(y, s)}\right) & \text { in } \Omega_{T}, \\ w_{(y, s)}=0 \quad \text { on } \Gamma_{T}^{D}, \quad \partial_{\nu} w_{(y, s)}=0 & \text { on } \Gamma_{T}^{N}, \\ w_{(y, s)}(x, 0)=0 \quad \text { for } x \in \Omega . & \end{cases}
$$

We call $w_{(y, s)}$ the reflected solution.

Proposition 2.5. For $(y, s),\left(y, s^{\prime}\right) \in(\Omega \backslash \bar{D})_{T}$ such that $s \neq s^{\prime}$, we have

$$
I\left(y, s^{\prime} ; y, s\right)=(k-1) \int_{D_{T}} \nabla\left(w_{(y, s)}+V_{(y, s)}\right) \cdot \nabla \Psi_{\left(y, s^{\prime}\right)} \mathrm{d} x \mathrm{~d} t
$$

Remark 2.6. The proofs of this proposition and the next theorem look similar to those in [4]. The differences of proofs happen because we are dealing with the mixed boundary value problem. 
Proof of Proposition 2.5. For simplicity, we drop the subscripts $(y, s)$ and $\left(y, s^{\prime}\right)$. By the definition of Neumann to Dirichlet map, we have

$$
\begin{aligned}
\int_{\Gamma_{T}^{N}} & \left(\Lambda_{\emptyset}-\Lambda_{D}\right)\left(\partial_{\nu} v^{j}\right) \partial_{\nu} \psi^{j}=\int_{\Gamma_{T}^{N}}\left(v^{j}-u^{j}\right) \partial_{\nu} \psi^{j}=-\int_{\Gamma_{T}^{N}} w^{j} \partial_{\nu} \psi^{j} \\
& =-\int_{\partial \Omega_{T}} w^{j} \partial_{\nu} \psi^{j}=-\int_{\Omega_{T}}\left(\nabla w^{j} \cdot \nabla \psi^{j}+w^{j} \Delta \psi^{j}\right) \\
& =-\int_{\Omega_{T}}\left[\left(\gamma-(k-1) \chi_{D}\right) \nabla w^{j} \cdot \nabla \psi^{j}+w^{j} \Delta \psi^{j}\right] \\
& =-\int_{\partial \Omega_{T}} \gamma \partial_{\nu} w^{j} \psi^{j}+\int_{\Omega_{T}}\left[\nabla \cdot\left(\gamma \nabla w^{j}\right) \psi^{j}+(k-1) \chi_{D} \nabla w^{j} \cdot \nabla \psi^{j}-\partial_{t} w^{j} \psi^{j}\right] \\
& =\int_{\Omega_{T}}\left[-(k-1) \nabla \cdot\left(\chi_{D} \nabla v^{j}\right) \psi^{j}+(k-1) \chi_{D} \nabla w^{j} \cdot \nabla \psi^{j}\right] \\
& =(k-1) \int_{D_{T}} \nabla\left(v^{j}+w^{j}\right) \cdot \nabla \psi^{j} .
\end{aligned}
$$

Let $U \subset \Omega_{(-\varepsilon, T+\varepsilon)}$ be an open set with property (R) in $\Omega_{(-\varepsilon, T+\varepsilon)},(y, s),\left(y, s^{\prime}\right) \notin$ $U$ and $\overline{D_{T}} \subset U$. Since $u^{j}=v^{j}+w^{j} \rightarrow V+w$ in $H^{1,0}(U)$, we have

$$
\int_{\Gamma_{T}^{N}}\left(\Lambda_{\emptyset}-\Lambda_{D}\right)\left(\partial_{\nu} v^{j}\right) \partial_{\nu} \psi^{j} \rightarrow(k-1) \int_{D_{T}} \nabla(V+w) \cdot \nabla \Psi \mathrm{d} x \mathrm{~d} t \quad \text { as } \quad j \rightarrow \infty .
$$

This completes the proof.

Moreover, we can derive the following representation for pre-indicator function.

Theorem 2.7. For $(y, s),\left(y, s^{\prime}\right) \in(\Omega \backslash \bar{D})_{T}$ with $s \neq s^{\prime}$, we have

$$
\begin{aligned}
I\left(y, s^{\prime} ; y, s\right)= & -\int_{\Gamma_{T}^{N}} w_{(y, s)} \partial_{\nu} \psi \mathrm{d} \sigma_{x} \mathrm{~d} t-\int_{\Omega_{s^{\prime}}}\left[\partial_{t} w_{(y, s)} G^{*}\left(\cdot, \cdot ; y, s^{\prime}\right)\right. \\
& \left.+\nabla w_{(y, s)} \cdot \nabla G^{*}\left(\cdot, \cdot ; y, s^{\prime}\right)\right] \mathrm{d} x \mathrm{~d} t .
\end{aligned}
$$

Proof. For $\varphi \in W\left(\Omega_{T}\right)$ with $\varphi(x, T)=0$ for all $x \in \Omega$ and $\left.\varphi\right|_{\Gamma_{T}^{D}}=0$, we have

$$
\begin{aligned}
& \int_{\Omega_{T}}\left(-w \partial_{t} \varphi+\nabla w \cdot \nabla \varphi\right)=\int_{\Omega_{T}}\left[\partial_{t} w \varphi+\left(\gamma-(k-1) \chi_{D}\right) \nabla w \cdot \nabla \varphi\right] \\
& =\int_{\Omega_{T}} \partial_{t} w \varphi+\int_{\partial \Omega_{T}} \gamma \partial_{\nu} w \varphi-\int_{\Omega_{T}}\left[\nabla \cdot(\gamma \nabla w) \varphi+(k-1) \chi_{D} \nabla w \cdot \nabla \varphi\right] \\
& =\int_{\Omega_{T}}(k-1) \nabla \cdot\left(\chi_{D} \nabla V\right) \varphi-(k-1) \int_{D_{T}} \nabla w \cdot \nabla \varphi \\
& =-(k-1) \int_{D_{T}} \nabla(V+w) \cdot \nabla \varphi .
\end{aligned}
$$


That is,

$$
\int_{\Omega_{T}}\left(-w \partial_{t} \varphi+\nabla w \cdot \nabla \varphi\right)=-(k-1) \int_{D_{T}} \nabla(V+w) \cdot \nabla \varphi
$$

for all $\varphi \in W\left(\Omega_{T}\right)$ with the properties $\varphi(x, T)=0$ and $\left.\varphi\right|_{\Gamma_{T}^{D}}=0$. Let us consider an open ball $B_{r}(y, s)$ of radius $r>0$ centered at $(y, s)$ in $\mathbb{R}^{n+1}$ and use $B_{r}$ for $B_{r}(0,0)$ for brevity. For $0<r<R<\infty$, let $\eta \in C_{0}^{\infty}\left(\mathbb{R}^{n+1}\right)$ be such that $0 \leq \eta \leq 1, \eta \equiv 1$ on $B_{r}, \operatorname{supp}(\eta) \subset B_{R}$, and $\left|\nabla_{x} \eta\right| \leq \frac{C}{R-r}$. For $\varepsilon>0$, let

$$
\eta_{\varepsilon}(x, t):=\eta\left(\frac{x-y}{\varepsilon}, \frac{t-s^{\prime}}{\varepsilon^{2}}\right) .
$$

Since $\left(y, s^{\prime}\right) \notin \overline{D_{T}}$, there is $\varepsilon_{0}$ such that $\eta_{\varepsilon}=0$ in $\overline{D_{T}}$ for all $0<\varepsilon<\varepsilon_{0}$. From now on we suppose that $\varepsilon<\varepsilon_{0}$ and fix an open set $E$ with $\operatorname{supp}\left(\eta_{\varepsilon}\right) \subset E \subset \bar{E} \subset$ $(\Omega \backslash \bar{D})_{T}$. We can further assume that $(y, s) \notin E$ by taking smaller $\varepsilon_{0}$. Substitute $\varphi=\left(1-\eta_{\varepsilon}\right) \Psi$ in (2.10). Then we have RHS of $(2.10)=-I\left(y, s^{\prime} ; y, s\right)$ and

$$
\operatorname{LHS} \text { of }(2.10)=\int_{\Omega_{T}}\left[-w \partial_{t} \Psi+\nabla w \cdot \nabla \Psi\right]+\int_{\Omega_{T}}\left[w \partial_{t}\left(\eta_{\varepsilon} \Psi\right)-\nabla w \cdot \nabla\left(\eta_{\varepsilon} \Psi\right)\right] \text {. }
$$

By the way, we have

$$
\begin{aligned}
\int_{\Omega_{T}}\left[-w \partial_{t} \Psi+\nabla w \cdot \nabla \Psi\right]= & \int_{\Omega_{T}}\left[-w \partial_{t} \psi+\nabla w \cdot \nabla \psi\right] \\
& +\int_{\Omega_{T}}\left[-w \partial_{t} G^{*}+\nabla w \cdot \nabla G^{*}\right] \\
= & \int_{\Omega_{T}}\left[-w \partial_{t} \psi-w \Delta \psi\right]+\int_{\partial \Omega_{T}} w \partial_{\nu} \psi \\
& +\int_{\Omega_{s^{\prime}}}\left[\partial_{t} w G^{*}+\nabla w \cdot \nabla G^{*}\right] \\
= & \int_{\Gamma_{T}^{N}} w \partial_{\nu} \psi+\int_{\Omega_{s^{\prime}}}\left[\partial_{t} w G^{*}+\nabla w \cdot \nabla G^{*}\right] .
\end{aligned}
$$

For the second term in (2.11), we have

$$
\begin{aligned}
\int_{\Omega_{T}}\left[w \partial_{t}\left(\eta_{\varepsilon} \Psi\right)-\nabla w \cdot \nabla\left(\eta_{\varepsilon} \Psi\right)\right]= & \int_{\Omega_{T}} w \partial_{t}\left(\eta_{\varepsilon} \psi\right)-\nabla w \cdot \nabla\left(\eta_{\varepsilon} \psi\right) \\
& +\int_{\Omega_{T}} w \partial_{t}\left(\eta_{\varepsilon} G^{*}\right)-\nabla w \cdot \nabla\left(\eta_{\varepsilon} G^{*}\right) \\
= & -\int_{\Omega_{T}}\left[\partial_{t} w \eta_{\varepsilon} \psi+\nabla w \cdot \nabla \eta_{\varepsilon} \psi+\nabla w \cdot \nabla \psi \eta_{\varepsilon}\right] \\
& -\int_{\Omega_{s^{\prime}}}\left[\partial_{t} w \eta_{\varepsilon} G^{*}+\nabla w \cdot \nabla \eta_{\varepsilon} G^{*}+\nabla w \cdot \nabla G^{*} \eta_{\varepsilon}\right] .
\end{aligned}
$$


Since $\psi, \nabla \psi, G^{*}, \nabla G^{*} \in L^{1}\left(\Omega_{T}\right)$ and $w \in C^{\infty}(E)$, we can see that each term below

$$
\int_{\Omega_{T}} \partial_{t} w \eta_{\varepsilon} \psi, \int_{\Omega_{T}} \nabla w \cdot \nabla \psi \eta_{\varepsilon}, \int_{\Omega_{s^{\prime}}} \partial_{t} w \eta_{\varepsilon} G^{*}, \int_{\Omega_{s^{\prime}}} \nabla w \cdot \nabla G^{*} \eta_{\varepsilon}
$$

goes to zero as $\varepsilon \rightarrow 0$. Set $Q_{r}:=\left\{(x, t) \in \mathbb{R}^{n+1}: \frac{|x-y|^{2}}{r^{2}}+\frac{\left(s^{\prime}-t\right)^{2}}{r^{4}} \leq 1\right\}$. Then, we have

$$
\begin{aligned}
\left|\int_{\Omega_{T}} \nabla w \cdot \nabla \eta_{\varepsilon} \psi\right| & =\left|\int_{E} \nabla w \cdot \nabla \eta_{\varepsilon} \psi\right| \\
& \leq \frac{C}{\varepsilon(R-r)}\|\psi\|_{L^{2}\left(\Omega_{T}\right)} \sup _{E}|\nabla w|\left[\int_{\left(Q_{\varepsilon R} \backslash Q_{\varepsilon r}\right) \cap \Omega_{T}} \mathrm{~d} x \mathrm{~d} t\right]^{1 / 2} \\
& =C^{\prime} \varepsilon^{-1} \varepsilon^{\frac{n}{2}+1}=C^{\prime \prime} \varepsilon^{\frac{n}{2}} \rightarrow 0 \quad \text { as } \varepsilon \rightarrow 0 .
\end{aligned}
$$

Finally, for the term

$$
\begin{aligned}
\int_{\Omega_{s^{\prime}}} \nabla w \cdot \nabla \eta_{\varepsilon} G^{*} & =\int_{E \cap \Omega_{s^{\prime}}} \nabla w \cdot \nabla \eta_{\varepsilon} G^{*} \\
& =\int_{E \cap \Omega_{s^{\prime}}} \nabla w \cdot \nabla \eta_{\varepsilon}\left(G^{*}-\Gamma^{*}\right)+\int_{E \cap \Omega_{s^{\prime}}} \nabla w \cdot \nabla \eta_{\varepsilon} \Gamma^{*} \\
& =I_{1}+I_{2},
\end{aligned}
$$

we get

$$
\left|I_{1}\right| \leq \frac{C}{\varepsilon(R-r)} \sup _{E}|\nabla w| \sup _{E \cap \Omega_{s^{\prime}}}\left|G^{*}-\Gamma^{*}\right| \int_{\left(Q_{\varepsilon R} \backslash Q_{\varepsilon r}\right) \cap \Omega_{s^{\prime}}} d x d t=C^{\prime} \varepsilon^{-1} \varepsilon^{n+2} \rightarrow 0
$$

as $\varepsilon \rightarrow 0$ and

$$
\begin{aligned}
\left|I_{2}\right| & \leq \sup _{E}|\nabla w| \frac{C}{\varepsilon(R-r)} \int_{\left(Q_{\varepsilon R} \backslash Q_{\varepsilon r}\right) \cap \Omega_{s^{\prime}}} \Gamma^{*} d x d t \\
& \leq C^{\prime} \varepsilon^{-1} \int_{\left(B_{R} \backslash B_{r}\right) \cap\left\{0<\tau<s^{\prime}\right\}} \frac{1}{\left(4 \pi \varepsilon^{2} \tau\right)^{n / 2}} \exp \left[-\frac{|\xi|^{2}}{4 \tau}\right] \varepsilon^{n+2} d \xi d \tau \\
& =C^{\prime \prime} \varepsilon \rightarrow 0 \quad \text { as } \varepsilon \rightarrow 0 .
\end{aligned}
$$

This completes the proof.

Finally, we have the following representation formula for the pre-indicator function in terms of the reflected solution. 
Theorem 2.8. For $(y, s),\left(y, s^{\prime}\right) \in(\Omega \backslash \bar{D})_{T}$ with $s \neq s^{\prime}$, we have

$$
I\left(y, s^{\prime} ; y, s\right)=-w_{(y, s)}\left(y, s^{\prime}\right)-\int_{\Gamma_{T}^{N}} w_{(y, s)}(x, t) \partial_{\nu} \Psi_{\left(y, s^{\prime}\right)}(x, t) d \sigma_{x} d t
$$

Proof. For an arbitrary positive constant $\varepsilon$, let $G_{\varepsilon}^{*}\left(x, t ; y, s^{\prime}\right)=G^{*}\left(x, t ; y, s^{\prime}+\varepsilon\right)$. To begin with, we prove the following:

$$
I\left(y, s^{\prime} ; y, s\right)=-\int_{\Gamma_{T}^{N}} w \partial_{\nu} \psi-\lim _{\varepsilon \rightarrow 0} \int_{\Omega_{s^{\prime}}}\left(\partial_{t} w G_{\varepsilon}^{*}+\nabla w \cdot \nabla G_{\varepsilon}^{*}\right) .
$$

Choose $\delta>0$ sufficiently small so that $B_{\delta}\left(y, s^{\prime}\right) \cap \overline{D_{T}}=\emptyset$. Let $\eta$ be a smooth cutoff function supported in $B_{\delta}\left(y, s^{\prime}\right)$ such that $\eta \equiv 1$ near $\left(y, s^{\prime}\right)$. Then by Theorem 2.7 we have

$$
\begin{aligned}
I\left(y, s^{\prime} ; y, s\right)+ & \int_{\Gamma_{T}^{N}} w \partial_{\nu} \psi+\int_{\Omega_{s^{\prime}}}\left(\partial_{t} w G_{\varepsilon}^{*}+\nabla w \cdot \nabla G_{\varepsilon}^{*}\right) \\
= & \int_{\Omega_{s^{\prime}}} \eta\left[\partial_{t} w\left(G_{\varepsilon}^{*}-G^{*}\right)+\nabla w \cdot \nabla\left(G_{\varepsilon}^{*}-G^{*}\right)\right] \\
& +\int_{\Omega_{s^{\prime}}}(1-\eta)\left[\partial_{t} w\left(G_{\varepsilon}^{*}-G^{*}\right)+\nabla w \cdot \nabla\left(G_{\varepsilon}^{*}-G^{*}\right)\right] \\
= & : I_{1}+I_{2} .
\end{aligned}
$$

Since $(1-\eta)\left(G_{\varepsilon}^{*}-G^{*}\right)=(1-\eta)\left[\left(G_{\varepsilon}^{*}-\Gamma_{\varepsilon}^{*}\right)-\left(G^{*}-\Gamma^{*}\right)+\left(\Gamma_{\varepsilon}^{*}-\Gamma^{*}\right)\right]$ is smooth in $\overline{\Omega_{s^{\prime}}}$ and tends to zero as $\varepsilon \rightarrow 0$ together with its derivatives, we have

$$
\begin{aligned}
\left|I_{2}\right| \leq & \left\|\partial_{t} w\right\|_{L^{2}\left(\left(0, s^{\prime}\right) ;\left(H^{1}(\Omega)\right)^{*}\right)}\left\|(1-\eta)\left(G_{\varepsilon}^{*}-G^{*}\right)\right\|_{H^{1,0}\left(\Omega_{s^{\prime}}\right)} \\
& +\left\|\nabla_{x} w\right\|_{L^{2}\left(\Omega_{s^{\prime}}\right)}\left\|(1-\eta) \nabla_{x}\left(G_{\varepsilon}^{*}-G^{*}\right)\right\|_{L^{2}\left(\Omega_{s^{\prime}}\right)},
\end{aligned}
$$

and hence $\left|I_{2}\right| \rightarrow 0$ as $\varepsilon \rightarrow 0$.

On the other hand, $w$ is smooth in $\overline{B_{\delta}\left(y, s^{\prime}\right)}$ and $\nabla_{x}\left(G_{\varepsilon}^{*}-G^{*}\right)$ converges to 0 in $L^{1}\left(B_{\delta}\left(y, s^{\prime}\right)\right)$ as $\varepsilon \rightarrow 0$. It then follows that

$$
\left|I_{1}\right| \leq C\left(\left\|G_{\varepsilon}^{*}-G^{*}\right\|_{L^{1}\left(B_{\delta}\left(y, s^{\prime}\right)\right)}+\left\|\nabla_{x}\left(G_{\varepsilon}^{*}-G^{*}\right)\right\|_{L^{1}\left(B_{\delta}\left(y, s^{\prime}\right)\right)}\right) \rightarrow 0
$$

as $\varepsilon \rightarrow 0$. 
Thus we have (2.13). By integration by parts, we get

$$
\begin{aligned}
\int_{\Omega_{s^{\prime}}}\left(\partial_{t} w G_{\varepsilon}^{*}+\nabla w \cdot \nabla G_{\varepsilon}^{*}\right)= & \int_{\Omega_{s^{\prime}}}\left(-w \partial_{t} G_{\varepsilon}^{*}-w \Delta G_{\varepsilon}^{*}\right) \\
& +\int_{\Omega} w\left(x, s^{\prime}\right) G_{\varepsilon}^{*}\left(x, s^{\prime} ; y, s^{\prime}\right) \mathrm{d} x+\int_{\partial \Omega_{s^{\prime}}} w \partial_{\nu} G_{\varepsilon}^{*} \\
= & \int_{\Omega} w\left(x, s^{\prime}\right) G^{*}\left(x, s^{\prime} ; y, s^{\prime}+\varepsilon\right)+\int_{\partial \Omega_{s^{\prime}}} w \partial_{\nu} G_{\varepsilon}^{*} \\
= & \int_{\Omega} w\left(x, s^{\prime}\right)\left(G^{*}\left(x, s^{\prime} ; y, s^{\prime}+\varepsilon\right)\right. \\
& \left.-\Gamma^{*}\left(x, s^{\prime} ; y, s^{\prime}+\varepsilon\right)\right) \\
& +\int_{\Omega} w\left(x, s^{\prime}\right) \Gamma^{*}\left(x, s^{\prime} ; y, s^{\prime}+\varepsilon\right)+\int_{\partial \Omega_{T}} w \partial_{\nu} G_{\varepsilon}^{*} .
\end{aligned}
$$

Note that for any $\varepsilon>0$

$$
G^{*}\left(x, s^{\prime} ; y, s^{\prime}+\varepsilon\right)-\Gamma^{*}\left(x, s^{\prime} ; y, s^{\prime}+\varepsilon\right)
$$

is $C^{\infty}\left(\overline{\Omega_{T}}\right)$ and goes to $G^{*}\left(x, s^{\prime} ; y, s^{\prime}\right)-\Gamma^{*}\left(x, s^{\prime} ; y, s^{\prime}\right)=0$ as $\varepsilon \rightarrow 0$. Since $\Gamma^{*}\left(x, s^{\prime} ; y, s^{\prime}+\varepsilon\right)=\Gamma(x, \varepsilon ; y, 0)$ and $\Gamma(x, \varepsilon ; y, 0)$ is the forward heat kernel, we have

$$
\lim _{\varepsilon \rightarrow 0} \int_{\Omega} w\left(x, s^{\prime}\right) \Gamma(x, \varepsilon ; y, 0) d x=w\left(y, s^{\prime}\right) .
$$

Therefore, we get

$$
\begin{aligned}
I\left(y, s^{\prime} ; y, s\right)= & -\int_{\Gamma_{T}^{N}} w_{(y, s)}(x, t) \partial_{\nu} \psi(x, t) \mathrm{d} \sigma_{x} \mathrm{~d} t-w_{(y, s)}\left(y, s^{\prime}\right) \\
& -\int_{\Gamma_{T}^{N}} w_{(y, s)}(x, t) \partial_{\nu_{x}} G^{*}\left(x, t ; y, s^{\prime}\right) \mathrm{d} \sigma_{x} \mathrm{~d} t \\
= & -w_{(y, s)}\left(y, s^{\prime}\right)-\int_{\Gamma_{T}^{N}} w_{(y, s)}(x, t) \partial_{\nu_{x}} \Psi_{\left(y, s^{\prime}\right)}(x, t) \mathrm{d} \sigma_{x} \mathrm{~d} t .
\end{aligned}
$$

Now we are ready to define the indicator function.

Definition 2.9. Let $C:=\{c(\lambda) ; 0 \leq \lambda \leq 1\}$ be a non-selfintersecting $C^{1}$ curve in $\bar{\Omega}$ which joins $c(0), c(1) \in \partial \Omega$. We call such a curve $C$ a needle. Then, for each $c(\lambda) \in \Omega$ and each fixed $s \in(0, T)$, we define the indicator function $J(c(\lambda), s)$ by

$$
J(c(\lambda), s):=\lim _{\epsilon \rightarrow 0} \liminf _{\delta \downarrow 0}\left|I\left(c(\lambda-\delta), s+\epsilon^{2} ; c(\lambda-\delta), s\right)\right|
$$

whenever the limit exists. 


$$
c(0)-\lambda \delta)
$$

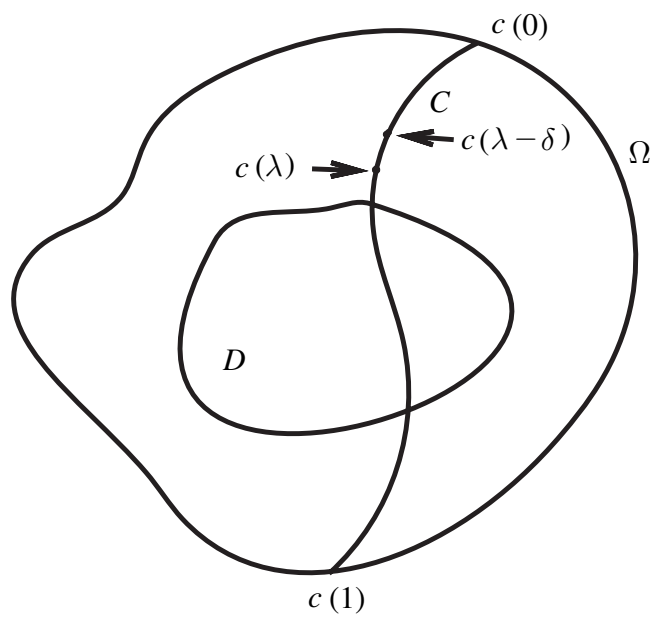

Theorem 2.10 (Main result). Let $C$ be given as in Definition 2.9 above. Then, for a fixed $s \in(0, T)$, we have the followings.

(i) If the curve $C$ is in $\Omega \backslash \bar{D}$ except $c(0)$ and $c(1)$, then $J(c(\lambda), s)<\infty$ for all $\lambda$, $0 \leq \lambda \leq 1$

(ii) If $\bar{C} \cap \bar{D} \neq \emptyset$, let $\lambda_{s}\left(0<\lambda_{s}<1\right)$ be such that $c\left(\lambda_{s}\right) \in \partial D$ and $c(\lambda) \in$ $\Omega \backslash \bar{D}\left(0<\lambda<\lambda_{s}\right)$. Namely, $c\left(\lambda_{s}\right)$ is the point at which $c$ first hits $\partial D$. We call $c\left(\lambda_{s}\right)$ the first hitting point. Then,

$$
\lambda_{s}=\sup \left\{0<\lambda<1 ; J\left(c\left(\lambda^{\prime}\right), s\right)<\infty \quad \text { for any } 0<\lambda^{\prime}<\lambda\right\} .
$$

Remark 2.11. Based on Theorem 2.10, we can in principle reconstruct $D$ as follows. We first draw many needles which could intersect with $\bar{D}$. Then, Theorem 2.10 says that by observing the behavior of the indicator function along each needle, we can extract the first hitting points for each needle. Then, if we consider some surface which is very close to these first hitting points, this surface will be an approximate reconstruction of $\partial D$. The numerical implementation of this reconstruction scheme has been already given for the two space dimensional case [18].

\section{Proof of Theorem 2.10}

The proof for the case $n=1$ is basically given in [4]. Since the rest of the cases can be handled in the same way, we will give the proof only for the case $n=3$.

Let $(y, s) \in \Omega_{T}$. First of all, by Theorem 2.8, we can analyze the behavior of the indicator function $J(c(\lambda), s)$ by the behavior of the reflected solution. If $y$ is not on the boundary of $D$, then $w_{(y, s)}$ is bounded due to the interior regularity 
estimate (see, for example [8]). So we only need to know the behavior of $w_{(y, s)}$ when $y$ approaches the boundary of $D$ along the curve $C$.

To start with, let $E(x, t ; y, s):=w_{(y, s)}(x, t)+V_{(y, s)}(x, t)$. Then, $E$ is obviously a fundamental solution for the operator $\mathcal{P}_{D}$. Further, let $C$ be as in Definition 2.9 .

Now let $P=c\left(\lambda_{0}\right) \in \partial D$ for some $\lambda_{0}$. Since $\partial D$ is $C^{1, \alpha}(0<\alpha<1)$, there is a $C^{1, \alpha}$ diffeomorphism $\Phi: \mathbb{R}^{3} \rightarrow \mathbb{R}^{3}$ which transforms $P$ to the origin $O$ in $\mathbb{R}^{3}$, $\Phi(D) \subset \mathbb{R}_{-}^{3}=\left\{x=\left(x_{1}, x_{2}, x_{3}\right) \in \mathbb{R}^{3} ; x_{3}<0\right\}$. This $\Phi$ is the same as that in the paper of G. Alessandrini and M. Di Cristo [1]. For reader's convenience, we will give the definition of $\Phi$ in details. To start with, let $P=\left(p_{1}, p_{2}, p_{3}\right)=\left(p^{\prime}, p_{3}\right) \in$ $\partial D$. Since $\partial D$ is of class $C^{1, \alpha}$, there exist positive constants $\bar{r}, L$ and a map $\phi$ under which we have

$$
D \cap B_{\bar{r}}(P)=\left\{x \in B_{\bar{r}}(P): x_{3}<p_{3}+\phi\left(x^{\prime}\right)\right\},
$$

where $\phi$ is a $C^{1, \alpha}$ function on $B_{\bar{r}}\left(p^{\prime}\right) \subset \mathbb{R}^{2}$ satisfying $\phi\left(p^{\prime}\right)=\left|\nabla \phi\left(p^{\prime}\right)\right|=0$ and $\|\phi\|_{C^{1, \alpha}\left(B_{\bar{r}}\left(p^{\prime}\right)\right)} \leq L \bar{r}$. Here the norm is defined as

$$
\|\phi\|_{C^{1, \alpha}\left(B_{\bar{r}}\left(p^{\prime}\right)\right)}=\|\phi\|_{L^{\infty}\left(B_{\bar{r}}\left(p^{\prime}\right)\right)}+\bar{r}\|\nabla \phi\|_{L^{\infty}\left(B_{\bar{r}}\left(p^{\prime}\right)\right)}+\bar{r}^{1+\alpha}|\nabla \phi|_{\alpha, B_{\bar{r}}\left(p^{\prime}\right)} .
$$

Then, let $\theta \in C^{\infty}(\mathbb{R})$ be such that $0 \leq \theta \leq 1, \theta(t)=1$ for $|t|<1, \theta(t)=0$ for $|t|>2$ and $\left|\frac{d \theta}{d t}\right| \leq 2$. We consider the following change of variables $\xi=\Phi(x)$ defined by

$$
\left\{\begin{array}{l}
\xi^{\prime}=x^{\prime}-p^{\prime} \\
\xi_{3}=x_{3}-p_{3}-\phi\left(x^{\prime}\right) \theta\left(\frac{\left|x^{\prime}-p^{\prime}\right|}{r_{1}}\right) \theta\left(\frac{x_{3}-p_{3}}{r_{1}}\right),
\end{array}\right.
$$

where $r_{1}=\frac{\bar{r}}{2} \min \left\{1,(8 L)^{-1 / \alpha}\right\}$. Then it can be easily verified that the Jacobian matrix of $\Phi$ at $P$ equals the identity matrix.

Let us proceed to our proof. Let $\Gamma_{-}$be the fundamental solution for the operator $\partial_{t}-\operatorname{div}\left(\left(1+(k-1) \chi_{-}\right) \nabla\right)$ in $\mathbb{R}^{4}$ with the characteristic function $\chi_{-}$of the space $\mathbb{R}_{-}^{3}$ such that $\Gamma_{-}(x, t ; y, s)=0$ for $t \leq s$. For this operator, we can explicitly compute the reflected solution $W(\xi, t ; \eta, s)$ with $\xi=\Phi(x), \eta=\Phi(y)$ of $\Gamma(\xi, t ; \eta, s)$ given as $W(\xi, t ; \eta, s)=\Gamma_{-}(\xi, t ; \eta, s)-\Gamma(\xi, t ; \eta, s)$. As a consequence, we can have estimate $W(\xi, t ; \eta, s)$ as $\xi=\eta \rightarrow O$ and $t \downarrow s$. Hence, in order to see the behavior of $w_{(y, s)}(x, t)$ as $x=y \rightarrow P$ and $t \downarrow s$, we compare $w_{(y, s)}(x, t)$ with $W(\Phi(x), t ; \Phi(y), s)$. More precisely, we decompose $w_{(y, s)}$ as follows:

$$
\begin{aligned}
w_{(y, s)}(x, t)= & E(x, t ; y, s)-V_{(y, s)}(x, t) \\
= & \left\{E(x, t ; y, s)-\Gamma_{-}(\Phi(x), t ; \Phi(y), s)\right\} \\
& +\left\{\Gamma_{-}(\Phi(x), t ; \Phi(y), s)-\Gamma(\Phi(x), t ; \Phi(y), s)\right\} \\
& +\{\Gamma(\Phi(x), t ; \Phi(y), s)-\Gamma(x, t ; y, s)\} \\
& +\left\{\Gamma(x, t ; y, s)-V_{(y, s)}(x, t)\right\} .
\end{aligned}
$$


From the definition of $V_{(y, s)}(x, t)$, we can see that it has the same singularity with $\Gamma(x, t ; y, s)$ and so the difference $\Gamma(x, t ; y, s)-V_{(y, s)}(x, t)$ is bounded. The third term is directly calculated because of the explicit definition of $\Gamma$ which is given in (2.1). In Lemma 3.6, we get the integral representation of the second term. This is obtained from Laplace transform and Fourier transform. This term is the dominant part in the behavior of $w_{(y, s)}(x, t)$.

Let $\varepsilon>0$ be given. We take $x=y=y(\delta)=c\left(\lambda_{0}-\delta\right) \in C \backslash \bar{D}$ so that $y(\delta) \rightarrow P(\delta \downarrow 0)$. Then, as $\delta \downarrow 0, \xi=\eta \rightarrow \Phi(P)=O$.

Remark 3.1. When $t>s$, the definition of $\Gamma$ gives us the fact

$$
\Gamma(y, t ; y, s)=\frac{1}{(4 \pi(t-s))^{3 / 2}} .
$$

So, the third parenthesis is zero if we take $x=y$ and $t=s+\varepsilon^{2}$.

As for the fourth term, we have the following lemma.

Lemma 3.2. We have

$$
\Gamma(x, t ; y, s)-V_{(y, s)}(x, t)
$$

is $C^{\infty}$ at $(y, s)$ and so bounded in some closed neighborhood of $(y, s)$.

Proof. Note that

$$
\Gamma(x, t ; y, s)-V_{(y, s)}(x, t)=\Gamma(x, t ; y, s)-G(x, t ; y, s)-v(x, t)
$$

and $\Gamma(x, t ; y, s)-G(x, t ; y, s) \in C^{\infty}\left(\overline{\Omega_{T}}\right)$. By the definition of the function $G^{*}$, we have, for $(x, t) \in \Omega_{T}$,

$$
\begin{aligned}
v(x, t)= & -\int_{\partial \Omega_{t}} v(z, \tau) \partial_{\nu_{z}} G^{*}(z, \tau ; x, t) \mathrm{d} \sigma_{z} \mathrm{~d} \tau \\
= & \int_{\partial \Omega_{t}} v(z, \tau) \partial_{\nu_{z}}\left(\Gamma^{*}-G^{*}\right)(z, \tau ; x, t) \mathrm{d} \sigma_{z} \mathrm{~d} \tau \\
& -\int_{\partial \Omega_{t}} v(z, \tau) \partial_{\nu_{z}} \Gamma^{*}(z, \tau ; x, t) \mathrm{d} \sigma_{z} \mathrm{~d} \tau .
\end{aligned}
$$

Since $\Gamma^{*}(x, t ; y, s)-G^{*}(x, t ; y, s)$ is $C^{\infty}\left(\overline{\Omega_{T}}\right)$, we can conclude that $v$ is smooth at $(y, s)$.

Next, we will estimate the remaining terms in (3.4) in the forthcoming lemmas:

$$
\begin{array}{r}
E\left(\Phi^{-1}(\xi), t ; \Phi^{-1}(\eta), s\right)-\Gamma_{-}(\xi, t ; \eta, s) \quad \text { in Lemma 3.3, } \\
\Gamma_{-}(\xi, t ; \eta, s)-\Gamma(\xi, t ; \eta, s) \quad \text { in Lemma 3.6. }
\end{array}
$$

To begin with, we put $\tilde{E}(\xi, t ; \eta, s):=E\left(\Phi^{-1}(\xi), t ; \Phi^{-1}(\eta), s\right)$. Then, we have the following. 
Lemma 3.3. If $y$ approaches the boundary of $D$, then there is a positive constant C such that

$$
\limsup _{\delta \downarrow 0}\left|\left(\tilde{E}-\Gamma_{-}\right)\left(\xi, s+\varepsilon^{2} ; \eta, s\right)\right| \leq C \varepsilon^{\alpha-3},
$$

where $C$ does not depend on $\varepsilon$.

Proof. Note that $\tilde{E}$ satisfies

$$
\left[\partial_{t}-\nabla_{\xi} \cdot\left(\left(1+(k-1) \chi_{-}\right) M(\xi) \nabla_{\xi}\right)\right] \tilde{E}(\xi, t ; \eta, s)=\delta(\xi-\eta) \delta(t-s)
$$

in $\mathbb{R}^{3} \times \mathbb{R}^{1}$, where $M(\xi)=J J^{T}$ with $J=\frac{\partial \xi}{\partial x}\left(\Phi^{-1}(\xi)\right)$ and $\tilde{E}(\xi, t ; \eta, s)=0$ for $t \leq s$. (Here, $J^{T}$ is the transpose matrix of $J$.) Then $\tilde{R}(\xi, t ; \eta, s):=\tilde{E}(\xi, t ; \eta, s)-$ $\Gamma_{-}(\xi, t ; \eta, s)$ satisfies

$$
\tilde{R}(\xi, t ; \eta, s)=\nabla_{\xi} \cdot\left(\left(1+(k-1) \chi_{-}\right)(M-I) \nabla_{\xi}\right) \tilde{E}(\xi, t ; \eta, s)
$$

in $\mathbb{R}^{3} \times \mathbb{R}^{1}$.

Now, let $\Gamma_{-}^{*}$ be the fundamental solution for $-\partial_{t}-\operatorname{div} \xi\left(\left(1+(k-1) \chi_{-}\right) \nabla_{\xi}\right)$ such that $\Gamma_{-}^{*}(\xi, t ; z, \tau)=0$ for $t \geq \tau$. Choose a ball $B_{r}(O)$ with a radius $r$ centered at the origin so that $\overline{\Phi(\Omega)} \subset B_{r}(O)$. Then, we have the following representation:

$$
\begin{aligned}
\tilde{R}(\xi, t ; \eta, s)= & \int_{s}^{t} \int_{B_{r}(O)}\left(1+(k-1) \chi_{-}\right)(I-M) \nabla_{z} \tilde{E}(z, \tau ; \eta, s) \\
& \cdot \nabla_{z} \Gamma_{-}^{*}(z, \tau ; \xi, t) \mathrm{d} z \mathrm{~d} \tau \\
& +\int_{s}^{t} \int_{\partial B_{r}(O)}\left(1+(k-1) \chi_{-}\right)\left[\frac{\partial}{\partial \nu_{z}} \tilde{R}(z, \tau ; \eta, s) \Gamma_{-}^{*}(z, \tau ; \xi, t)\right. \\
& \left.-\tilde{R}(z, \tau ; \eta, s) \frac{\partial}{\partial \nu_{z}} \Gamma_{-}^{*}(z, \tau ; \xi, t)\right] \mathrm{d} \sigma_{z} \mathrm{~d} \tau .
\end{aligned}
$$

Note that the integration (3.7) is finite by the choice of $B_{r}(O)$.

For analyzing the behavior of $\tilde{R}$, we need to know the estimate of gradients of $\tilde{E}$ and $\Gamma_{-}^{*}$. That is we need the estimates:

$$
\begin{aligned}
& \left|\nabla_{z} \tilde{E}(z, \tau ; \eta, s)\right| \leq c_{1}(\tau-s)^{-2} \exp \left[-\frac{|z-\eta|^{2}}{c_{2}(\tau-s)}\right], \\
& \left|\nabla_{z} \Gamma_{-}^{*}(z, \tau ; \xi, t)\right| \leq c_{3}(t-\tau)^{-2} \exp \left[-\frac{|z-\xi|^{2}}{c_{4}(t-\tau)}\right]
\end{aligned}
$$

for some constants $c_{i}>0(1 \leq i \leq 4)$. For the derivation of these estimates, we gave some comment and references right after the end of the present proof.

To proceed further, we use the estimate $|M(z)-I| \leq C|z|^{\alpha}(|z|<r)$ for some constant $C>0$, because $\partial D$ is $C^{1, \alpha}$. Note that as $y$ approaches the $\partial D$ (that is, 
$\delta \downarrow 0), \xi=\eta \rightarrow O$. By using the Fatou lemma, the lim $\sup _{\delta \downarrow 0}$ of the absolute value of the integration (3.6) can be bounded from above by a constant multiple of the following:

$$
\int_{s}^{t} \int_{|z|<r}|z|^{\alpha}\left|\nabla_{z} \tilde{E}(z, \tau ; O, s)\right|\left|\nabla_{z} \Gamma_{-}^{*}(z, \tau ; O, t)\right| d z d \tau=: F .
$$

Now let us substitute $t=s+\varepsilon^{2}$. From (3.8) and (3.9), we have

$$
\begin{aligned}
F= & \int_{s}^{s+\varepsilon^{2}} \int_{|z|<r}|z|^{\alpha}\left|\nabla_{z} \tilde{E}(z, \tau ; O, s)\right|\left|\nabla_{z} \Gamma_{-}^{*}(z, \tau ; O, t)\right| \mathrm{d} z \mathrm{~d} \tau \\
\leq & C_{1} \int_{s}^{s+\varepsilon^{2}} \int_{|z|<r}|z|^{\alpha}(\tau-s)^{-2}\left(s+\varepsilon^{2}-\tau\right)^{-2} \\
& \cdot \exp \left[-\frac{\varepsilon^{2}|z|^{2}}{C_{2}(\tau-s)\left(s+\varepsilon^{2}-\tau\right)}\right] \mathrm{d} z \mathrm{~d} \tau \\
\leq & C_{3} \varepsilon^{-6} \int_{0}^{1} \int_{\mathbb{R}^{3}}|z|^{\alpha}(\mu(1-\mu))^{-2} \exp \left[-\frac{|z|^{2}}{C_{2} \varepsilon^{2} \mu(1-\mu)}\right] \mathrm{d} z \mathrm{~d} \mu \\
\leq & C_{4} \varepsilon^{\alpha-3} \int_{0}^{1} \int_{\mathbb{R}^{3}}(\mu(1-\mu))^{\frac{\alpha-1}{2}}|\zeta|^{\alpha} \exp \left[-\frac{|\zeta|^{2}}{C_{2}}\right] \mathrm{d} \zeta \mathrm{d} \mu \\
\leq & C_{5} \varepsilon^{\alpha-3}
\end{aligned}
$$

with some constants $C_{i}>0(1 \leq i \leq 5)$. Thus we can conclude that $F \leq C \varepsilon^{\alpha-3}$. Finally, we obtain

$$
\limsup _{\delta \downarrow 0}\left|\tilde{R}\left(\xi, s+\varepsilon^{2} ; \eta, s\right)\right| \leq C \varepsilon^{\alpha-3}
$$

for some positive constant $C$ which does not depend on $\varepsilon$.

The estimates (3.8) and (3.9) are proved in [5]. For the reader's convenience, we write down the results proved there.

Let $D$ be a bounded domain in $\mathbb{R}^{n}, n=2,3$, with a $C^{1, \alpha}$ boundary $(0<$ $\alpha<1$ ) and let $D_{m}, 1 \leq m \leq L$, be a finite number of disjoint subdomains of $D$, each with a $C^{1, \alpha}$ boundary. Furthermore, suppose that $\bar{D}=\cup_{m=1}^{L} \bar{D}_{m}$. Let $A^{(m)} \in C^{\mu}\left(\bar{D}_{m}\right)(0<\mu<1)$ be a symmetric, positive definite matrix-valued function, and define

$$
A(x)=A^{(m)}(x), \quad x \in D_{m}, 1 \leq m \leq L .
$$

Under these assumptions, authors in [5] proved the following Theorem 3.4 and Proposition 3.5. 
Theorem 3.4 ([5]). Let $0<r<T, \overline{r Q} \subset D$ and $u \in W\left(r Q \times\left(-r^{2}, r^{2}\right)\right)$ be a solution of $\left(\partial_{t}-\nabla \cdot A \nabla\right) u=0$ in $r Q \times\left(-r^{2}, r^{2}\right)$. Then, there exists a constant $c>0$ such that for any $0<\rho<r / 2$ and $(x, t) \in(r-2 \rho) Q \times\left(-r^{2}+4 \rho^{2}, r^{2}\right)$, we have

$$
\left\|\nabla_{x} u\right\|_{L^{\infty}\left(\rho Q(x) \times\left(-\rho^{2}+t, t\right)\right)} \leq \frac{c}{\rho^{n / 2+2}}\|u\|_{L^{2}\left(2 \rho Q(x) \times\left(-4 \rho^{2}+t, t\right)\right)},
$$

where $Q(x):=\left\{y=\left(y_{1}, \cdots, y_{n}\right) \in \mathbb{R}^{n}:\left|x_{i}-y_{i}\right|<1,1 \leq i \leq n\right\}$ with $x=\left(x_{1}, \cdots, x_{n}\right)$ and $Q:=Q(0)$.

It is well known that there exists a fundamental solution $\Gamma(x, t ; y, s)$ of the operator $\partial_{t}-\nabla \cdot A \nabla$ with the estimate

$$
\Gamma(x, t ; y, s) \leq \frac{C}{[4 \pi(t-s)]^{n / 2}} e^{-\frac{|x-y|^{2}}{C(t-s)}} \chi_{[s, \infty)} \quad(t, s \in \mathbb{R}, t>s \text {, a.e. } x, y \in D),
$$

which is positive for $t>s$, where $C>0$ is a constant which depends only on $A, n$ and $\chi_{[s, \infty)}$ is the characteristic function of $[s, \infty)$ (See [2]). As an application of Theorem 3.4, they derived the estimate of $\nabla_{x} \Gamma$ by combining the scaling argument of Di Cristo-Vessella [6], that is,

Proposition 3.5 ([5]). Let $\Gamma(x, t ; y, s)$ be the previous fundamental solution. Then, there exists a constant $C>0$ depending only on $A$ and $n$ such that

$$
\left|\nabla_{x} \Gamma(x, t ; y, s)\right| \leq \frac{C}{(t-s)^{\frac{n+1}{2}}} e^{-\frac{|x-y|^{2}}{C(t-s)}}
$$

for any $t, s \in \mathbb{R}, t>s$ and almost every $x, y \in D$.

Now put

$$
W(\xi, t ; \eta, s):=\Gamma_{-}(\xi, t ; \eta, s)-\Gamma(\xi, t ; \eta, s)
$$

Let us denote $W(\xi, t ; \eta, s)$ for $\pm \xi_{3}>0$ by $W^{ \pm}(\xi, t ; \eta, s)$. Then, we have the following lemma.

Lemma 3.6. If $y$ approaches the boundary of $D$, then there is a nonzero constant $C$ which does not depend on $\varepsilon$ such that

$$
\lim _{\delta \downarrow 0} W^{+}\left(\xi, s+\varepsilon^{2} ; \eta, s\right)=C \varepsilon^{-3} .
$$

Because the proof is little bit tedious, we will explain the outline of it in advance. $W$ satisfies (3.10) which can be expressed as a transmission problem (3.11). At first, we apply Laplace transform to (3.11) with respect to $t$ and then apply Fourier transform to it with respect to $\xi^{\prime}=\left(\xi_{1}, \xi_{2}\right)$. Then, we can get the ordinary differential equation with respect to $\xi_{3}$. This ordinary differential equation is solved explicitly. 
To this explicitly expressed solution, we apply inverse Laplace transform and then inverse Fourier transform. From these elementary calculations, we finally get the integral representation of the solution (See (3.13)). It can be justified that this is the solution to the original problem (3.11) we want. (You can find this justification in appendix.) So, $W$ is given as an inverse Laplace-Fourier transform with respect to the Fourier variable $\zeta^{\prime}$ of $\xi^{\prime}$ and Laplace variable $\tau$ of $t$. We first reduce the inverse Laplace transform to an integral along a segment joining the two branch cuts of integrand. Then, the integration with respect to $\zeta^{\prime}$ can be computed explicitly if we let $\xi=\eta \rightarrow 0$ and $t=s+\varepsilon^{2}$. At this point, $W$ is given as $\varepsilon^{-3}$ times an integral over $(0,1)$ which turns out to be a nonzero constant. Now, let us start the proof of Lemma.

Proof. We first note that

$$
\left\{\begin{array}{l}
\partial_{t} \Gamma_{-}(\xi, t ; \eta, s)-\nabla_{\xi} \cdot\left(1+(k-1) \chi_{-}\right) \nabla_{\xi} \Gamma_{-}(\xi, t ; \eta, s)=\delta(\xi-\eta) \delta(t-s) \\
\partial_{t} \Gamma(\xi, t ; \eta, s)-\triangle_{\xi} \Gamma(\xi, t ; \eta, s)=\delta(\xi-\eta) \delta(t-s)
\end{array}\right.
$$

in $\mathbb{R}^{3} \times \mathbb{R}^{1}$.

Then, for any $\varphi$ in $C_{0}^{\infty}\left(\mathbb{R}^{3} \times(0, \infty)\right)$, we have

$$
\begin{aligned}
& \int_{0}^{\infty} \int_{\mathbb{R}^{3}}\left[\partial_{t} W \varphi+\left(1+(k-1) \chi_{-}\right) \nabla W \cdot \nabla \varphi\right] \mathrm{d} \xi \mathrm{d} t \\
& =\int_{0}^{\infty} \int_{\mathbb{R}^{3}}\left[\partial_{t} \Gamma-\varphi+\left(1+(k-1) \chi_{-}\right) \nabla \Gamma_{-} \cdot \nabla \varphi\right] \mathrm{d} \xi \mathrm{d} t \\
& \quad-\int_{0}^{\infty} \int_{\mathbb{R}^{3}}\left[\partial_{t} \Gamma \varphi+\left(1+(k-1) \chi_{-}\right) \nabla \Gamma \cdot \nabla \varphi\right] \mathrm{d} \xi \mathrm{d} t \\
& =\varphi(\eta, s)-\varphi(\eta, s)-\int_{0}^{\infty} \int_{\mathbb{R}^{3}}(k-1) \chi_{-} \nabla \Gamma \cdot \nabla \varphi \mathrm{d} \xi \mathrm{d} t \\
& =-\int_{0}^{\infty} \int_{\mathbb{R}^{3}}(k-1) \chi_{-} \nabla \Gamma \cdot \nabla \varphi \mathrm{d} \xi \mathrm{d} t .
\end{aligned}
$$

Thus, $W$ satisfies

$$
\begin{aligned}
& \partial_{t} W(\xi, t ; \eta, s)-\nabla_{\xi} \cdot\left(\left(1+(k-1) \chi_{-}\right) \nabla_{\xi} W(\xi, t ; \eta, s)\right) \\
& =(k-1) \nabla_{\xi} \cdot\left(\chi_{-} \nabla_{\xi} \Gamma(\xi, t ; \eta, s)\right) .
\end{aligned}
$$

Let us analyze the behavior of $W$. Let $\hat{\Gamma}$ be the Laplace transform of $\Gamma$ with respect to $t$, that is,

$$
\hat{\Gamma}(\xi, \tau ; \eta, s)=\int_{0}^{\infty} e^{-t \tau} \Gamma(\xi, t ; \eta, s) \mathrm{d} t .
$$

Thus, $\hat{\Gamma}$ satisfies

$$
\tau \hat{\Gamma}(\xi, \tau ; \eta, s)-\triangle_{\xi} \hat{\Gamma}(\xi, \tau ; \eta, s)=e^{-\tau s} \delta(\xi-\eta) \quad \text { in } \quad \mathbb{R}^{3} .
$$


Now, we denote $\Gamma$ for $\pm\left(\xi_{3}-\eta_{3}\right)>0$ by $\Gamma^{ \pm}$. For $\varphi \in C_{0}^{\infty}\left(\mathbb{R}^{3}\right)$, we have

$$
\begin{aligned}
0= & \int_{\mathbb{R}^{3}}\left[\tau \hat{\Gamma} \varphi+\nabla \hat{\Gamma} \cdot \nabla \varphi-e^{-\tau s} \delta(\xi-\eta) \varphi\right] \mathrm{d} \xi \\
= & \int_{\mathbb{R}^{3}} \tau \hat{\Gamma} \varphi d \xi+\int_{\left\{\xi_{3}-\eta_{3}>0\right\}} \nabla \hat{\Gamma}^{+} \cdot \nabla \varphi \mathrm{d} \xi+\int_{\left\{\xi_{3}-\eta_{3}<0\right\}} \nabla \hat{\Gamma}^{-} \cdot \nabla \varphi \mathrm{d} \xi \\
& -\int_{\left\{\xi_{3}-\eta_{3}=0\right\}} e^{-\tau s} \delta\left(\xi^{\prime}-\eta^{\prime}\right) \varphi\left(\xi^{\prime}, \eta_{3}\right) \mathrm{d} \xi^{\prime} \\
= & \int_{\mathbb{R}^{3}} \tau \hat{\Gamma} \varphi \mathrm{d} \xi-\int_{\left\{\xi_{3}-\eta_{3}=0\right\}} \frac{\partial \hat{\Gamma}^{+}}{\partial \xi_{3}} \varphi \mathrm{d} \xi^{\prime}-\int_{\left\{\xi_{3}-\eta_{3}>0\right\}} \Delta \hat{\Gamma}^{+} \varphi \mathrm{d} \xi \\
& +\int_{\left\{\xi_{3}-\eta_{3}=0\right\}} \frac{\partial \hat{\Gamma}^{-}}{\partial \xi_{3}} \varphi \mathrm{d} \xi^{\prime}-\int_{\left\{\xi_{3}-\eta_{3}<0\right\}} \Delta \hat{\Gamma}^{-} \varphi \mathrm{d} \xi \\
& -\int_{\left\{\xi_{3}-\eta_{3}=0\right\}} e^{-\tau s} \delta\left(\xi^{\prime}-\eta^{\prime}\right) \varphi\left(\xi^{\prime}, \eta_{3}\right) \mathrm{d} \xi^{\prime} \\
= & \int_{\left\{\xi_{3}-\eta_{3}>0\right\}}\left[\tau \hat{\Gamma}^{+}-\Delta \hat{\Gamma}^{+}\right] \varphi \mathrm{d} \xi+\int_{\left\{\xi_{3}-\eta_{3}<0\right\}}\left[\tau \hat{\Gamma}^{-}-\Delta \hat{\Gamma}^{-}\right] \varphi \mathrm{d} \xi \\
& +\int_{\left\{\xi_{3}-\eta_{3}=0\right\}}\left[\frac{\partial \hat{\Gamma}^{-}}{\partial \xi_{3}}-\frac{\partial \hat{\Gamma}^{+}}{\partial \xi_{3}}-e^{-\tau s} \delta\left(\xi^{\prime}-\eta^{\prime}\right)\right] \varphi \mathrm{d} \xi^{\prime} .
\end{aligned}
$$

Therefore, we have the following transmission problem:

$$
\left\{\begin{array}{l}
\triangle \hat{\Gamma}^{ \pm}-\tau \hat{\Gamma}^{ \pm}=0 \quad \text { in }\left\{ \pm\left(\xi_{3}-\eta_{3}\right)>0\right\} \\
\hat{\Gamma}^{+}-\hat{\Gamma}^{-}=0 \quad \text { on }\left\{\xi_{3}-\eta_{3}=0\right\}, \\
\frac{\partial \hat{\Gamma}^{+}}{\partial \xi_{3}}-\frac{\partial \hat{\Gamma}^{-}}{\partial \xi_{3}}=-e^{-\tau s} \delta\left(\xi^{\prime}-\eta^{\prime}\right) \quad \text { on }\left\{\xi_{3}-\eta_{3}=0\right\} .
\end{array}\right.
$$

Let $\phi^{ \pm}$be the Fourier transforms of $\hat{\Gamma}^{ \pm}$for $\xi^{\prime}=\left(\xi_{1}, \xi_{2}\right)$. From now on, we use $\zeta^{\prime}=\left(\zeta_{1}, \zeta_{2}\right)$ to denote the Fourier variable associated with $\xi^{\prime}$. Then, we have

$$
\left\{\begin{array}{l}
\frac{\partial^{2} \phi^{ \pm}}{\partial \xi_{3}^{2}}-\left(\left|\zeta^{\prime}\right|^{2}+\tau\right) \phi^{ \pm}=0 \quad \text { in }\left\{ \pm\left(\xi_{3}-\eta_{3}\right)>0\right\} \\
\phi^{+}-\phi^{-}=0 \quad \text { on }\left\{\xi_{3}-\eta_{3}=0\right\} \\
\frac{\partial \phi^{+}}{\partial \xi_{3}}-\frac{\partial \phi^{-}}{\partial \xi_{3}}=-e^{-\tau s} e^{-i \eta^{\prime} \cdot \zeta^{\prime}} \quad \text { on }\left\{\xi_{3}-\eta_{3}=0\right\} .
\end{array}\right.
$$

This is an ordinary differential equation in $\mathbb{R}^{3}$ with transmission boundary conditions on $\left\{\xi_{3}-\eta_{3}=0\right\}$. So, proper calculations give us

$$
\left\{\begin{array}{l}
\phi^{+}\left(\zeta^{\prime}, \xi_{3}\right)=\frac{1}{2 \Theta\left(\zeta^{\prime}, \tau\right)} \exp \left(-\tau s-i \eta^{\prime} \cdot \zeta^{\prime}-\left(\xi_{3}-\eta_{3}\right) \Theta\left(\zeta^{\prime}, \tau\right)\right) \\
\phi^{-}\left(\zeta^{\prime}, \xi_{3}\right)=\frac{1}{2 \Theta\left(\zeta^{\prime}, \tau\right)} \exp \left(-\tau s-i \eta^{\prime} \cdot \zeta^{\prime}+\left(\xi_{3}-\eta_{3}\right) \Theta\left(\zeta^{\prime}, \tau\right)\right),
\end{array}\right.
$$


where $\Theta^{2}\left(\zeta^{\prime}, \tau\right)=\left|\zeta^{\prime}\right|^{2}+\tau$ and real part $\Re \Theta$ of $\Theta>0$. Thus we obtain the representation of $\hat{\Gamma}^{ \pm}$:

$$
\begin{aligned}
\hat{\Gamma}^{ \pm}(\xi, \tau ; \eta, s) & =\frac{1}{(2 \pi)^{2}} \int_{\mathbb{R}^{2}} e^{i \xi^{\prime} \cdot \zeta^{\prime}} \phi^{ \pm}\left(\zeta^{\prime}, \xi_{3}\right) \mathrm{d} \zeta^{\prime} \\
& =\frac{1}{8 \pi^{2}} \int_{\mathbb{R}^{2}} \frac{1}{\Theta\left(\zeta^{\prime}, \tau\right)} e^{-\tau s+i\left(\xi^{\prime}-\eta^{\prime}\right) \cdot \zeta^{\prime} \mp\left(\xi_{3}-\eta_{3}\right) \Theta\left(\zeta^{\prime}, \tau\right)} \mathrm{d} \zeta^{\prime} .
\end{aligned}
$$

Note that $W$ satisfies the following transmission problem:

$$
\left\{\begin{array}{l}
\partial_{t} W^{+}-\Delta W^{+}=0 \quad \text { in }\left\{\xi_{3}>0\right\}, \\
\partial_{t} W^{-}-k \Delta W^{-}=(k-1) \Delta \Gamma^{-} \quad \text { in }\left\{\xi_{3}<0\right\}, \\
W^{+}-W^{-}=0 \quad \text { on }\left\{\xi_{3}=0\right\}, \\
\frac{\partial W^{+}}{\partial \xi_{3}}-k \frac{\partial W^{-}}{\partial \xi_{3}}=(k-1) \frac{\partial \Gamma^{-}}{\partial \xi_{3}} \quad \text { on }\left\{\xi_{3}=0\right\}, \\
W^{+}(\xi, 0)=0 \text { in }\left\{\xi_{3}>0\right\}, \quad W^{-}(\xi, 0)=0 \text { in }\left\{\xi_{3}<0\right\} .
\end{array}\right.
$$

Let $\hat{W}^{ \pm}$be the Laplace transform of $W^{ \pm}$with respect to $t$. Then we get

$$
\left\{\begin{array}{l}
\tau \hat{W}^{+}-\triangle \hat{W}^{+}=0 \quad \text { in }\left\{\xi_{3}>0\right\}, \\
\tau\left(\hat{W}^{-}+\hat{\Gamma}^{-}\right)-k \triangle\left(\hat{W}^{-}+\hat{\Gamma}^{-}\right)=0 \quad \text { in }\left\{\xi_{3}<0\right\}, \\
\hat{W}^{+}-\hat{W}^{-}=0 \quad \text { on }\left\{\xi_{3}=0\right\}, \\
\frac{\partial \hat{W}^{+}}{\partial \xi_{3}}-k \frac{\partial \hat{W}^{-}}{\partial \xi_{3}}=(k-1) \frac{\partial \hat{\Gamma}^{-}}{\partial \xi_{3}} \quad \text { on }\left\{\xi_{3}=0\right\} .
\end{array}\right.
$$

By the same method that we used to obtain the representation of $\hat{\Gamma}^{ \pm}$, we can obtain the representation of $\hat{W}^{ \pm}$. In other words, $\hat{W}^{+}$and $\hat{W}^{-}$have the representations:

$$
\begin{aligned}
\hat{W}^{+}(\xi, \tau ; \eta, s)= & \frac{1}{8 \pi^{2}} \int_{\mathbb{R}^{2}} \frac{\Theta-k \Theta_{k}}{\Theta\left(\Theta+k \Theta_{k}\right)} \exp \left[-s \tau+i\left(\xi^{\prime}-\eta^{\prime}\right) \cdot \zeta^{\prime}-\left(\xi_{3}+\eta_{3}\right) \Theta\right] \mathrm{d} \zeta^{\prime}, \\
\hat{W}^{-}(\xi, \tau ; \eta, s)= & \frac{1}{4 \pi^{2}} \int_{\mathbb{R}^{2}} \frac{1}{\Theta+k \Theta_{k}} \exp \left[-s \tau+i\left(\xi^{\prime}-\eta^{\prime}\right) \cdot \zeta^{\prime}+\xi_{3} \Theta_{k}-\eta_{3} \Theta\right] \mathrm{d} \zeta^{\prime} \\
& -\frac{1}{8 \pi^{2}} \int_{\mathbb{R}^{2}} \frac{1}{\Theta} \exp \left[-s \tau+i\left(\xi^{\prime}-\eta^{\prime}\right) \cdot \zeta^{\prime}+\left(\xi_{3}-\eta_{3}\right) \Theta\right] \mathrm{d} \zeta^{\prime},
\end{aligned}
$$

where

$$
\begin{aligned}
& \Theta^{2}:=\Theta\left(\zeta^{\prime}, \tau\right)^{2}=\left|\zeta^{\prime}\right|^{2}+\tau, \quad \Re \Theta>0, \\
& \Theta_{k}^{2}:=\Theta_{k}\left(\zeta^{\prime}, \tau\right)^{2}=\left|\zeta^{\prime}\right|^{2}+\tau / k, \quad \Re \Theta_{k}>0 .
\end{aligned}
$$

Therefore, by the inversion formula for the Laplace transform, we have

$$
\begin{aligned}
W^{+}(\xi, t ; \eta, s) & =\frac{1}{2 \pi i} \int_{\sigma-i \infty}^{\sigma+i \infty} e^{t \tau} \hat{W}^{+}(\xi, \tau ; \eta, s) \mathrm{d} \tau \\
& =\frac{1}{4 \pi^{2}} \int_{\mathbb{R}^{2}} e^{i\left(\xi^{\prime}-\eta^{\prime}\right) \cdot \zeta^{\prime}} h\left(\zeta^{\prime}, t\right) \mathrm{d} \zeta^{\prime},
\end{aligned}
$$


where $\sigma>0$ and

$$
h\left(\zeta^{\prime}, t\right)=\frac{1}{4 \pi i} \int_{\sigma-i \infty}^{\sigma+i \infty} \frac{\Theta-k \Theta_{k}}{\Theta\left(\Theta+k \Theta_{k}\right)} e^{(t-s) \tau-\left(\xi_{3}+\eta_{3}\right) \Theta} \mathrm{d} \tau
$$

(in appendix, we show that $W^{ \pm}$obtained from this derivation satisfy (3.11).)

Since the case $k<1$ can be handled in the same way as the case $k>1$, we assume $k>1$ from now on. Performing a contour integration with appropriate branch cuts for the multi-valued functions $\Theta$ and $\Theta_{k}$ in (3.14)(see appendix), we have

$$
\begin{aligned}
h\left(\zeta^{\prime}, t\right)= & \frac{\sqrt{k-1}}{4 \pi} \int_{0}^{1} \frac{\left|\zeta^{\prime}\right|}{\sqrt{r}} \exp \left[-(t-s)\left|\zeta^{\prime}\right|^{2}(k r-r+1)\right] \\
\times & {\left[\frac{\sqrt{r}-i \sqrt{k(1-r)}}{\sqrt{r}+i \sqrt{k(1-r)}} \exp \left\{i\left(\xi_{3}+\eta_{3}\right) \sqrt{(k-1) r}\left|\zeta^{\prime}\right|\right\}\right.} \\
& \left.+\frac{\sqrt{r}+i \sqrt{k(1-r)}}{\sqrt{r}-i \sqrt{k(1-r)}} \exp \left\{-i\left(\xi_{3}+\eta_{3}\right) \sqrt{(k-1) r}\left|\zeta^{\prime}\right|\right\}\right] \mathrm{d} r .
\end{aligned}
$$

Hence, $W^{+}(\xi, t ; \eta, s)$ becomes

$$
\begin{aligned}
W^{+}(\xi, t ; \eta, s) & =\frac{\sqrt{k-1}}{16 \pi^{3}} \int_{0}^{1} \frac{1}{\sqrt{r}}\left[\frac{\sqrt{r}-i \sqrt{k(1-r)}}{\sqrt{r}+i \sqrt{k(1-r)}} I_{+}+\frac{\sqrt{r}+i \sqrt{k(1-r)}}{\sqrt{r}-i \sqrt{k(1-r)}} I_{-}\right] \mathrm{d} r \\
& =\frac{\sqrt{k-1}}{16 \pi^{3}} \int_{0}^{1}\left[\frac{(k+1) r-k}{\sqrt{r}(r+k(1-r))}\left(I_{+}+I_{-}\right)-2 i \frac{\sqrt{k(1-r)}}{r+k(1-r)}\left(I_{+}-I_{-}\right)\right] \mathrm{d} r,
\end{aligned}
$$

where

$$
I_{ \pm}=\int_{\mathbb{R}^{2}}\left|\zeta^{\prime}\right| e^{i\left(\xi^{\prime}-\eta^{\prime}\right) \cdot \zeta^{\prime}} \exp \left[-(t-s)\left|\zeta^{\prime}\right|^{2}(k r-r+1) \pm i\left(\xi_{3}+\eta_{3}\right) \sqrt{(k-1) r}\left|\zeta^{\prime}\right|\right] \mathrm{d} \zeta^{\prime} .
$$

Now let $\delta \downarrow 0$ and set $t=s+\varepsilon^{2}$. Then, $\xi=\eta \rightarrow O$ and $(t-s)(k r-r+1)>0$. Thus we have

$$
\begin{aligned}
\lim _{\delta \downarrow 0} I_{ \pm} & =\int_{\mathbb{R}^{2}}\left|\zeta^{\prime}\right| \exp \left[-\varepsilon^{2}(k r-r+1)\left|\zeta^{\prime}\right|^{2}\right] \mathrm{d} \zeta^{\prime} \\
& =2 \pi \int_{0}^{\infty} \rho^{2} e^{-(k r-r+1) \varepsilon^{2} \rho^{2}} \mathrm{~d} \rho \\
& =\frac{1}{2}\left(\frac{\pi}{k r-r+1}\right)^{\frac{3}{2}} \varepsilon^{-3}
\end{aligned}
$$

Therefore we have

$$
\lim _{\delta \downarrow 0} W^{+}\left(\xi, s+\varepsilon^{2} ; \eta, s\right)=\frac{\sqrt{k-1}}{16 \pi \sqrt{\pi} \varepsilon^{3}} H,
$$


where

$$
H=\int_{0}^{1} \frac{(k+1) r-k}{\sqrt{r}(r+k(1-r))(k r-r+1)^{3 / 2}} \mathrm{~d} r .
$$

In order to estimate $|H|$ from above and below, we note the following estimates:

$$
-k \leq(k+1) r-k \leq 1,1 \leq r+k-k r \leq k, 1 \leq k r-r+1 \leq k .
$$

Then we have

$$
|H| \leq \int_{0}^{1} \frac{k}{\sqrt{r}} \mathrm{~d} r=2 k<\infty .
$$

To show that $H \neq 0$, we write $H$ as the sum of the following terms

$$
\begin{aligned}
& H_{1}=\int_{0}^{\frac{k}{k+1}} \frac{(k+1) r-k}{\sqrt{r}(r+k(1-r))(k r-r+1)^{3 / 2}} \mathrm{~d} r, \\
& H_{2}=\int_{\frac{k}{k+1}}^{1} \frac{(k+1) r-k}{\sqrt{r}(r+k(1-r))(k r-r+1)^{3 / 2}} \mathrm{~d} r .
\end{aligned}
$$

Then, we have

$$
\begin{aligned}
-H_{1} & =\int_{0}^{\frac{k}{k+1}} \frac{k-(k+1) r}{\sqrt{r}(r+k(1-r))(k r-r+1)^{3 / 2}} \mathrm{~d} r \\
& \geq \int_{0}^{\frac{k}{k+1}} \frac{k-(k+1) r}{\left(\frac{k}{k+1}\right)^{1 / 2} k\left(\frac{k^{2}+1}{k+1}\right)^{3 / 2}} \mathrm{~d} r=\frac{k^{1 / 2}(k+1)}{2\left(k^{2}+1\right)^{3 / 2}},
\end{aligned}
$$

and for $\mathrm{H}_{2}$ we have

$$
H_{2} \leq \int_{\frac{k}{k+1}}^{1} \frac{(k+1) r-k}{\left(\frac{k}{k+1}\right)^{1 / 2}\left(\frac{k^{2}+1}{k+1}\right)^{\frac{3}{2}}} \mathrm{~d} r=\frac{k+1}{2 k^{1 / 2}\left(k^{2}+1\right)^{3 / 2}} .
$$

Thus, we have

$$
\begin{aligned}
H=H_{1}+H_{2} & \leq-\frac{k^{1 / 2}(k+1)}{2\left(k^{2}+1\right)^{3 / 2}}+\frac{k+1}{2 k^{1 / 2}\left(k^{2}+1\right)^{3 / 2}} \\
& =-\frac{(k+1)(k-1)}{2 k^{1 / 2}\left(k^{2}+1\right)^{3 / 2}}<0,
\end{aligned}
$$

since we assumed that $k>1$. This completes the proof.

By combining results in this section, we conclude that

$$
\liminf _{\delta \downarrow 0}\left|w_{(y(\delta), s)}\left(y(\delta), s+\varepsilon^{2}\right)\right| \geq C \varepsilon^{-3},
$$

for some positive constant $C$ which does not depend on $\varepsilon$. That is, the reflected solution blows up as the point approaches the boundary of the inhomogeneity. Furthermore, to get the blow up property we only use the perturbation in time. 


\section{A. Appendix}

In this appendix, we will provide the proofs for the two facts which we need in Section 3. They are

(i) $W^{ \pm}$solve the transmission problem (3.11),

(ii) formula (3.15).

\section{Proof of the fact that $W^{ \pm}$are the solution to (3.11).}

To verify this, we apply the method used in [13]. Note that

$$
\begin{aligned}
W^{+}(\xi, t) & =\frac{1}{2 \pi i} \int_{\sigma-i \infty}^{\sigma+i \infty} e^{t \tau} \hat{W}^{+}(\xi, \tau) \mathrm{d} \tau, \\
W^{-}(\xi, t) & =\frac{1}{2 \pi i} \int_{\sigma-i \infty}^{\sigma+i \infty} e^{t \tau} \hat{W}^{-}(\xi, \tau) \mathrm{d} \tau,
\end{aligned}
$$

where

$$
\begin{aligned}
\hat{W}^{+}(\xi, \tau)= & \frac{1}{8 \pi^{2}} \int_{\mathbb{R}^{2}} \frac{\Theta-k \Theta_{k}}{\Theta\left(\Theta+k \Theta_{k}\right)} \exp \left[-s \tau+i\left(\xi^{\prime}-\eta^{\prime}\right) \cdot \zeta^{\prime}-\left(\xi_{3}+\eta_{3}\right) \Theta\right] \mathrm{d} \zeta^{\prime} \\
= & : \frac{1}{8 \pi^{2}} \int_{\mathbb{R}^{2}} f\left(\xi, \zeta^{\prime}, \tau\right) \mathrm{d} \zeta^{\prime} \\
\hat{W}^{-}(\xi, \tau)= & \frac{1}{4 \pi^{2}} \int_{\mathbb{R}^{2}} \frac{1}{\Theta+k \Theta_{k}} \exp \left[-s \tau+i\left(\xi^{\prime}-\eta^{\prime}\right) \cdot \zeta^{\prime}+\xi_{3} \Theta_{k}-\eta_{3} \Theta\right] \mathrm{d} \zeta^{\prime} \\
& -\frac{1}{8 \pi^{2}} \int_{\mathbb{R}^{2}} \frac{1}{\Theta} \exp \left[-s \tau+i\left(\xi^{\prime}-\eta^{\prime}\right) \cdot \zeta^{\prime}+\left(\xi_{3}-\eta_{3}\right) \Theta\right] \mathrm{d} \zeta^{\prime} \\
= & : F_{1}+F_{2} .
\end{aligned}
$$

Let $\tau=\sigma+i \theta$, where $\tau \in \Pi_{\sigma_{0}}:=\left\{\sigma+i \theta \in \mathbb{C}: \sigma \geq \sigma_{0}^{\prime}>\sigma_{0}>0\right\}$. Then,

$$
\Theta=\left[\left|\zeta^{\prime}\right|^{2}+\tau\right]^{1 / 2}=\left[\left(\left|\zeta^{\prime}\right|^{2}+\sigma\right)^{2}+\theta^{2}\right]^{1 / 4} e^{i \alpha / 2}
$$

where $\alpha=\tan ^{-1} \frac{\theta}{\left|\zeta^{\prime}\right|^{2}+\sigma}$. Since $\sigma>0,\left(\left|\zeta^{\prime}\right|^{2}+\sigma\right)^{2}+\theta^{2} \geq \max \left\{\left|\zeta^{\prime}\right|^{4},|\tau|^{2}\right\}$. Thus

$$
\Re \Theta \geq C \max \left\{\left|\zeta^{\prime}\right|,|\tau|^{1 / 2}\right\}
$$

for some positive constant $C$. Moreover, since $\left(\left|\zeta^{\prime}\right|^{2}+\sigma\right)^{2}+\theta^{2} \leq 2\left(\left|\zeta^{\prime}\right|^{4}+|\tau|^{2}\right)$, we have

$$
\left(\left|\zeta^{\prime}\right|^{2}+\sigma\right)^{2}+\theta^{2} \leq\left\{\begin{array}{lll}
4\left|\zeta^{\prime}\right|^{4} & \text { if } & \left|\zeta^{\prime}\right| \geq|\tau|^{1 / 2} \\
4|\tau|^{2} & \text { if } & \left|\zeta^{\prime}\right|<|\tau|^{1 / 2}
\end{array}\right.
$$


In another words, we have

$$
\Re \Theta \leq\left\{\begin{array}{lll}
C_{1}\left|\zeta^{\prime}\right| & \text { if } & \left|\zeta^{\prime}\right| \geq|\tau|^{1 / 2} \\
C_{1}|\tau|^{1 / 2} & \text { if } & \left|\zeta^{\prime}\right|<|\tau|^{1 / 2}
\end{array}\right.
$$

for some positive constant $C_{1}$. So we will divide the domain of integration $\mathbb{R}^{2}$ in two parts $\left\{\zeta^{\prime} \in \mathbb{R}^{2}:\left|\zeta^{\prime}\right| \geq \sqrt{|\tau|}\right\}$ and $\left\{\zeta^{\prime} \in \mathbb{R}^{2}:\left|\zeta^{\prime}\right|<\sqrt{|\tau|}\right\}$. For $\Theta_{k}=$ $\sqrt{\left|\zeta^{\prime}\right|^{2}+\tau / k}$, we can get similar results as those for $\Theta$.

At first, let us consider $\hat{W}^{+}$. Let $\xi_{3} \in[a, b] \subset(0, \infty)$. Then,

$$
\Re\left[\left(\xi_{3}+\eta_{3}\right) \Theta\right]=\left(\xi_{3}+\eta_{3}\right) \Re \Theta \geq C \max \left\{\left(a+\eta_{3}\right)\left|\zeta^{\prime}\right|, \quad\left(a+\eta_{3}\right)|\tau|^{1 / 2}\right\}
$$

for some positive constant $C$. Thus, by (A.2), (A.3) and change of variables, we have

$$
\begin{aligned}
\left|\hat{W}^{+}(\xi, \tau)\right| \leq & C_{0} \int_{\left|\zeta^{\prime}\right| \geq|\tau|^{1 / 2}} \frac{\left|\zeta^{\prime}\right|}{\left|\zeta^{\prime}\right|^{2}} \exp \left[-s \sigma_{0}-C\left(a+\eta_{3}\right)\left|\zeta^{\prime}\right|\right] \mathrm{d} \zeta^{\prime} \\
& +C_{0} \int_{\left|\zeta^{\prime}\right|<|\tau|^{1 / 2}} \frac{|\tau|^{1 / 2}}{|\tau|} \exp \left[-s \sigma_{0}-C\left(a+\eta_{3}\right)|\tau|^{1 / 2}\right] \mathrm{d} \zeta^{\prime} \\
= & C_{0}^{\prime} e^{-s \sigma_{0}} \int_{|\tau|^{1 / 2}}^{\infty} e^{-C\left(a+\eta_{3}\right) r} \mathrm{~d} r+\frac{C_{0}^{\prime}}{|\tau|^{1 / 2}} e^{-s \sigma_{0}-C\left(a+\eta_{3}\right)|\tau|^{1 / 2}} \int_{0}^{|\tau|^{1 / 2}} r \mathrm{~d} r \\
= & C^{\prime}\left[\frac{1}{a+\eta_{3}}+|\tau|^{1 / 2}\right] e^{-s \sigma_{0}-C\left(a+\eta_{3}\right)|\tau|^{1 / 2}} .
\end{aligned}
$$

For $j \in\{1,2\}$, we have

$$
\partial_{\xi_{j}} f=i \zeta_{j} \frac{\Theta-k \Theta_{k}}{\Theta\left(\Theta+k \Theta_{k}\right)} \exp \left[-s \tau+i\left(\xi^{\prime}-\eta^{\prime}\right) \cdot \zeta^{\prime}-\left(\xi_{3}+\eta_{3}\right) \Theta\right] .
$$

Thus, we can find some constant $C_{0}$ such that

$$
\left|\partial_{\xi_{j}} f\right| \leq\left\{\begin{array}{lll}
C_{0}\left|\zeta^{\prime}\right| \frac{\left|\zeta^{\prime}\right|}{\left|\zeta^{\prime}\right|^{2}} e^{-s \sigma_{0}-C\left(a+\eta_{3}\right)\left|\zeta^{\prime}\right|} & \text { if } & \left|\zeta^{\prime}\right| \geq|\tau|^{1 / 2} \\
C_{0}\left|\zeta^{\prime}\right| \frac{|\tau|^{1 / 2}}{|\tau|} e^{-s \sigma_{0}-C\left(a+\eta_{3}\right)|\tau|^{1 / 2}} & \text { if } & \left|\zeta^{\prime}\right|<|\tau|^{1 / 2}
\end{array}\right.
$$

Therefore, we obtain

$$
\begin{aligned}
& \int_{\mathbb{R}^{2}}\left|\partial_{\xi_{j}} f\right| \mathrm{d} \zeta^{\prime} \\
& \leq C_{0} \int_{\left|\zeta^{\prime}\right| \geq|\tau|^{1 / 2}} e^{-s \sigma_{0}-C\left(a+\eta_{3}\right)\left|\zeta^{\prime}\right|} \mathrm{d} \zeta^{\prime}+\frac{C_{0}}{|\tau|^{1 / 2}} e^{-s \sigma_{0}-C\left(a+\eta_{3}\right)|\tau|^{1 / 2}} \int_{\left|\zeta^{\prime}\right|<|\tau|^{1 / 2}}\left|\zeta^{\prime}\right| \mathrm{d} \zeta^{\prime} \\
& =C^{\prime}\left[\frac{1}{\left(a+\eta_{3}\right)^{2}}+\frac{|\tau|^{1 / 2}}{a+\eta_{3}}+|\tau|\right] e^{-s \sigma_{0}-C\left(a+\eta_{3}\right)|\tau|^{1 / 2}} .
\end{aligned}
$$


When $j, l \in\{1,2\}$, we have

$$
\partial_{\xi_{j}} \partial_{\xi_{l}} f=\left(i \zeta_{j}\right)\left(i \zeta_{l}\right) \frac{\Theta-k \Theta_{k}}{\Theta\left(\Theta+k \Theta_{k}\right)} \exp \left[-s \tau+i\left(\xi^{\prime}-\eta^{\prime}\right) \cdot \zeta^{\prime}-\left(\xi_{3}+\eta_{3}\right) \Theta\right]
$$

Hence,

$$
\begin{aligned}
& \int_{\mathbb{R}^{2}}\left|\partial_{\xi_{j}} \partial_{\xi_{l}} f\right| \mathrm{d} \zeta^{\prime} \\
& \leq C_{0} \int_{\left|\zeta^{\prime}\right| \geq|\tau|^{1 / 2}}\left|\zeta^{\prime}\right|^{2} \frac{\left|\zeta^{\prime}\right|}{\left|\zeta^{\prime}\right|^{2}} e^{-s \sigma_{0}-C\left(a+\eta_{3}\right)\left|\zeta^{\prime}\right|} \mathrm{d} \zeta^{\prime} \\
& \quad+C_{0} \int_{|\zeta|<|\tau|^{1 / 2}}\left|\zeta^{\prime}\right|^{2} \frac{|\tau|^{1 / 2}}{|\tau|} e^{-s \sigma_{0}-C\left(a+\eta_{3}\right)|\tau|^{1 / 2}} \mathrm{~d} \zeta^{\prime} \\
& =C^{\prime}\left[\frac{1}{\left(a+\eta_{3}\right)^{3}}+\frac{|\tau|^{1 / 2}}{\left(a+\eta_{3}\right)^{2}}+\frac{|\tau|}{a+\eta_{3}}+|\tau|^{3 / 2}\right] e^{-s \sigma_{0}-C\left(a+\eta_{3}\right)|\tau|^{1 / 2}} .
\end{aligned}
$$

Similarly, we get

$$
\begin{aligned}
& \int_{\mathbb{R}^{2}}\left|\partial_{\xi_{3}} f\right| \mathrm{d} \zeta^{\prime} \leq C^{\prime}\left[\frac{1}{\left(a+\eta_{3}\right)^{2}}+\frac{|\tau|^{1 / 2}}{a+\eta_{3}}+|\tau|\right] e^{-s \sigma_{0}-C\left(a+\eta_{3}\right)|\tau|^{1 / 2}} \\
& \int_{\mathbb{R}^{2}}\left|\partial_{\xi_{3}}^{2} f\right| \mathrm{d} \zeta^{\prime} \leq C^{\prime}\left[\frac{1}{\left(a+\eta_{3}\right)^{3}}+\frac{|\tau|^{1 / 2}}{\left(a+\eta_{3}\right)^{2}}+\frac{|\tau|}{a+\eta_{3}}+|\tau|^{3 / 2}\right] e^{-s \sigma_{0}-C\left(a+\eta_{3}\right)|\tau|^{1 / 2}}
\end{aligned}
$$

Consequently, for $\xi \in \mathbb{R}^{2} \times[a, b] \subset \mathbb{R}^{2} \times(0, \infty)$ and $\tau \in \Pi_{\sigma_{0}}$, we have

$$
\begin{gathered}
\left|\hat{W}^{+}(\xi, \tau)\right| \leq C^{\prime}\left[\frac{1}{a+\eta_{3}}+|\tau|^{1 / 2}\right] e^{-s \sigma_{0}-C\left(a+\eta_{3}\right)|\tau|^{1 / 2}}, \\
\left|\partial_{\xi_{j}} \hat{W}^{+}(\xi, \tau)\right| \leq C^{\prime}\left[\frac{1}{\left(a+\eta_{3}\right)^{2}}+\frac{|\tau|^{1 / 2}}{a+\eta_{3}}+|\tau|\right] e^{-s \sigma_{0}-C\left(a+\eta_{3}\right)|\tau|^{1 / 2}}, \\
\left|\partial_{\xi_{j}}^{2} \hat{W}^{+}(\xi, \tau)\right| \leq C^{\prime}\left[\frac{1}{\left(a+\eta_{3}\right)^{3}}+\frac{|\tau|^{1 / 2}}{\left(a+\eta_{3}\right)^{2}}+\frac{|\tau|}{a+\eta_{3}}+|\tau|^{3 / 2}\right] e^{-s \sigma_{0}-C\left(a+\eta_{3}\right)|\tau|^{1 / 2}}
\end{gathered}
$$

for $j=1,2,3$.

Next, we consider $\hat{W}^{-}$. Let $\xi_{3} \in[c, d] \subset(-\infty, 0)$. Then, there is a positive constant $C$ such that

$$
\mathfrak{R}\left[\xi_{3} \Theta_{k}-\eta_{3} \Theta\right]=\xi_{3} \mathfrak{R} \Theta_{k}-\eta_{3} \Re \Theta \leq C \min \left\{\left(d-\eta_{3}\right)\left|\zeta^{\prime}\right|, \quad\left(d-\eta_{3}\right)|\tau|^{1 / 2}\right\} .
$$


Note that $\eta_{3}>0$. If we carry out similar calculations as we did for $\hat{W}^{+}$, we have

$$
\begin{aligned}
\left|\hat{W}^{-}(\xi, \tau)\right| & \leq C^{\prime}\left[\frac{1}{\eta_{3}-d}+|\tau|^{1 / 2}\right] e^{-s \sigma_{0}-C\left(\eta_{3}-d\right)|\tau|^{1 / 2}}, \\
\left|\partial_{\xi_{j}} \hat{W}^{-}(\xi, \tau)\right| & \leq C^{\prime}\left[\frac{1}{\left(\eta_{3}-d\right)^{2}}+\frac{|\tau|^{1 / 2}}{\eta_{3}-d}+|\tau|\right] e^{-s \sigma_{0}-C\left(\eta_{3}-d\right)|\tau|^{1 / 2}}, \\
\left|\partial_{\xi_{j}}^{2} \hat{W}^{-}(\xi, \tau)\right| & \leq C^{\prime}\left[\frac{1}{\left(\eta_{3}-d\right)^{3}}+\frac{|\tau|^{1 / 2}}{\left(\eta_{3}-d\right)^{2}}+\frac{|\tau|}{\eta_{3}-d}+|\tau|^{3 / 2}\right] e^{-s \sigma_{0}-C\left(\eta_{3}-d\right)|\tau|^{1 / 2}}
\end{aligned}
$$

for $j=1,2,3$.

Now, we show that $W^{ \pm}$satisfy (3.11). Note that $\hat{\Gamma}^{-}(\xi, \tau)=-F_{2}(\xi, \tau)$. Since we know

$$
\int_{\sigma_{0}}^{\infty} r^{m} e^{-\alpha \sqrt{r}} d r<\infty \quad \text { for } m=0, \frac{1}{2}, 1, \frac{3}{2}, \quad \alpha>0,
$$

if we apply this fact to $G(\xi, \tau)=\hat{W}^{ \pm}, \partial_{\xi_{j}} \hat{W}^{ \pm}, \partial_{\xi_{j}}^{2} \hat{W}^{ \pm}, \hat{\Gamma}^{-}, \partial_{\xi_{j}} \hat{\Gamma}^{-}, \partial_{\xi_{j}}^{2} \hat{\Gamma}^{-}$, then we have

$$
\int_{\sigma_{0}}^{\infty}|G(\xi, \tau)| d|\tau|<\infty
$$

Note that $\hat{W}^{ \pm}$satisfy (3.12). Taking into account (A.4) for $W^{+}$which allow to put the operator $\partial_{t}-\Delta \xi$ in the integrand of the improper integral, we have

$$
\begin{aligned}
\left(\partial_{t}-\Delta_{\xi}\right) W^{+}(\xi, t) & =\left(\partial_{t}-\Delta_{\xi}\right) \frac{1}{2 \pi i} \int_{\sigma-i \infty}^{\sigma+i \infty} e^{t \tau} \hat{W}^{+}(\xi, \tau) \mathrm{d} \tau \\
& =\frac{1}{2 \pi i} \int_{\sigma-i \infty}^{\sigma+i \infty} e^{t \tau}\left(\tau-\Delta_{\xi}\right) \hat{W}^{+}(\xi, \tau) \mathrm{d} \tau=0 .
\end{aligned}
$$

Similarly, from the facts (A.5), we have

$$
\begin{aligned}
\left(\partial_{t}-k \Delta_{\xi}\right) W^{-}(\xi, t) & =\left(\partial_{t}-k \Delta_{\xi}\right) \frac{1}{2 \pi i} \int_{\sigma-i \infty}^{\sigma+i \infty} e^{t \tau} \hat{W}^{-}(\xi, \tau) \mathrm{d} \tau \\
& =\frac{1}{2 \pi i} \int_{\sigma-i \infty}^{\sigma+i \infty} e^{t \tau}\left(\tau-k \Delta_{\xi}\right) \hat{W}^{-}(\xi, \tau) \mathrm{d} \tau \\
& =\frac{1}{2 \pi i} \int_{\sigma-i \infty}^{\sigma+i \infty} e^{t \tau}\left(k \Delta_{\xi}-\tau\right) \hat{\Gamma}^{-}(\xi, \tau) \mathrm{d} \tau \\
& =\left(k \Delta_{\xi}-\partial_{t}\right) \frac{1}{2 \pi i} \int_{\sigma-i \infty}^{\sigma+i \infty} e^{t \tau} \hat{\Gamma}^{-}(\xi, \tau) \mathrm{d} \tau \\
& =\left(k \Delta_{\xi}-\partial_{t}\right) \Gamma^{-}(\xi, t)=(k-1) \Delta_{\xi} \Gamma^{-}(\xi, t) .
\end{aligned}
$$


To verify that $W^{ \pm}$satisfy the boundary condition, it is necessary to take the limit $\xi_{3} \rightarrow 0$ in the integrand of (A.1). (For precise explanation, see [13, page 465].)

$$
\begin{aligned}
& \lim _{\xi_{3} \rightarrow 0^{+}} W^{+}(\xi, t)=\frac{1}{2 \pi i} \int_{\sigma-i \infty}^{\sigma+i \infty} e^{t \tau} \lim _{\xi_{3} \rightarrow 0^{+}} \hat{W}^{+}(\xi, \tau) \mathrm{d} \tau \\
& =\frac{1}{2 \pi i} \int_{\sigma-i \infty}^{\sigma+i \infty} e^{t \tau} \lim _{\xi_{3} \rightarrow 0^{-}} \hat{W}^{-}(\xi, \tau) \mathrm{d} \tau=\lim _{\xi_{3} \rightarrow 0^{-}} W^{-}(\xi, t) . \\
& \lim _{\xi_{3} \rightarrow 0^{+}} \frac{\partial}{\partial \xi_{3}} W^{+}(\xi, t)=\lim _{\xi_{3} \rightarrow 0^{+}} \frac{1}{2 \pi i} \int_{\sigma-i \infty}^{\sigma+i \infty} e^{t \tau} \frac{\partial}{\partial \xi_{3}} \hat{W}^{+}(\xi, \tau) \mathrm{d} \tau \\
& =\frac{1}{2 \pi i} \int_{\sigma-i \infty}^{\sigma+i \infty} e^{t \tau} \lim _{\xi_{3} \rightarrow 0^{+}} \frac{\partial}{\partial \xi_{3}} \hat{W}^{+}(\xi, \tau) \mathrm{d} \tau \\
& =\frac{1}{2 \pi i} \int_{\sigma-i \infty}^{\sigma+i \infty} e^{t \tau} \lim _{\xi_{3} \rightarrow 0^{-}}\left(k \frac{\partial}{\partial \xi_{3}} \hat{W}^{-}(\xi, \tau)+(k-1) \frac{\partial}{\partial \xi_{3}} \hat{\Gamma}^{-}(\xi, \tau)\right) \mathrm{d} \tau \\
& =\lim _{\xi_{3} \rightarrow 0^{-}} \frac{1}{2 \pi i} \int_{\sigma-i \infty}^{\sigma+i \infty} e^{t \tau}\left(k \frac{\partial}{\partial \xi_{3}} \hat{W}^{-}(\xi, \tau)+(k-1) \frac{\partial}{\partial \xi_{3}} \hat{\Gamma}^{-}(\xi, \tau)\right) \mathrm{d} \tau \\
& =\lim _{\xi_{3} \rightarrow 0^{-}}\left[k \frac{\partial}{\partial \xi_{3}} W^{-}(\xi, \tau)+(k-1) \frac{\partial}{\partial \xi_{3}} \Gamma^{-}(\xi, \tau)\right] .
\end{aligned}
$$

To verify that $W^{ \pm}$satisfy the initial conditions, we consider, for $N>0$, the integral

$$
W_{N}^{+}(\xi, t):=\frac{1}{2 \pi i} \int_{\gamma} e^{t \tau} \hat{W}^{+}(\xi, \tau) d \tau,
$$

where $\gamma=-\Gamma_{N}+C_{N}$ is a closed contour given as Figure A.1.

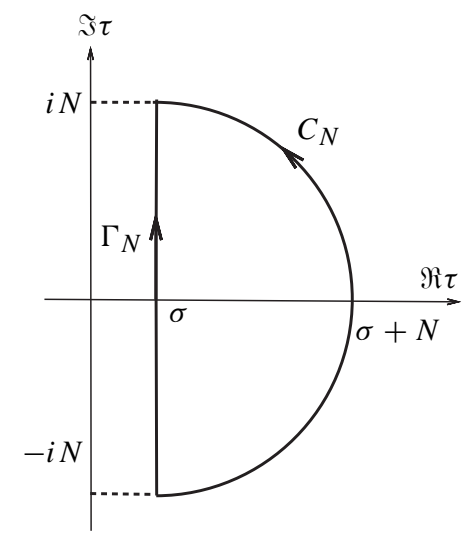

Figure A.1. Contour $\gamma$.

By Cauchy's theorem, we have

$$
\frac{1}{2 \pi i} \int_{\gamma} \hat{W}^{+}(\xi, \tau) \mathrm{d} \tau=0
$$


Note that

$$
\left|\hat{W}^{+}(\xi, \tau)\right| \leq C^{\prime}\left[\frac{1}{a+\eta_{3}}+|\tau|^{1 / 2}\right] e^{-s \sigma_{0}-C\left(a+\eta_{3}\right)|\tau|^{1 / 2}},
$$

for $a, \eta_{3}, C>0$. Thus

$$
\begin{aligned}
\int_{C_{N}}\left|\hat{W}^{+}(\xi, \tau)\right| d \tau & \leq C^{\prime \prime} \int_{-\frac{\pi}{2}}^{\frac{\pi}{2}}\left(\frac{1}{a+\eta_{3}}+\sqrt{2\left(\sigma^{2}+N^{2}\right)}\right) e^{-s \sigma_{0}-C\left(a+\eta_{3}\right) N^{1 / 2}} N \mathrm{~d} \theta \\
& =C^{\prime \prime \prime} N\left(\frac{1}{a+\eta_{3}}+\sqrt{2\left(\sigma^{2}+N^{2}\right)}\right) e^{-s \sigma_{0}-C\left(a+\eta_{3}\right) N^{1 / 2}} \\
& \rightarrow 0 \quad \text { as } N \rightarrow \infty
\end{aligned}
$$

Here we used

$$
N \leq|\tau|=\left|\sigma+N e^{i \theta}\right| \leq \sqrt{2\left(\sigma^{2}+N^{2}\right)} .
$$

Hence we have

$$
\begin{aligned}
\lim _{t \rightarrow 0} W^{+}(\xi, t) & =\lim _{N \rightarrow \infty} \frac{1}{2 \pi i} \int_{\Gamma_{N}} \hat{W}^{+}(\xi, \tau) \mathrm{d} \tau \\
& =\lim _{N \rightarrow \infty} \frac{1}{2 \pi i} \int_{C_{N}} \hat{W}^{+}(\xi, \tau) \mathrm{d} \tau=0 .
\end{aligned}
$$

By the same argument, we can also get

$$
\lim _{t \rightarrow 0} W^{-}(\xi, t)=0 .
$$

\section{Proof of formula (3.15).}

$W^{+}$was given by formula (3.13):

$$
W^{+}(\xi, t)=\frac{1}{(2 \pi)^{2}} \int_{\mathbb{R}^{2}} e^{i\left(\xi^{\prime}-\eta^{\prime}\right) \cdot \zeta^{\prime}} h\left(\zeta^{\prime}, t\right) \mathrm{d} \zeta^{\prime},
$$

where

$$
h\left(\zeta^{\prime}, t\right)=\frac{1}{4 \pi i} \int_{\sigma-i \infty}^{\sigma+i \infty} \frac{\Theta-k \Theta_{k}}{\Theta\left(\Theta+k \Theta_{k}\right)} e^{(t-s) \tau-\left(\xi_{3}+\eta_{3}\right) \Theta} \mathrm{d} \tau,
$$

with $\sigma>0$ and

$$
\begin{aligned}
& \Theta^{2}:=\Theta\left(\zeta^{\prime}, \tau\right)^{2}=\left|\zeta^{\prime}\right|^{2}+\tau, \quad \Re \Theta>0, \\
& \Theta_{k}^{2}:=\Theta_{k}\left(\zeta^{\prime}, \tau\right)^{2}=\left|\zeta^{\prime}\right|^{2}+\tau / k, \quad \Re \Theta_{k}>0 .
\end{aligned}
$$

We denote the integrand in $h\left(\zeta^{\prime}, t\right)$ by $g(\tau)$. That is,

$$
\begin{aligned}
g(\tau)= & \frac{\sqrt{\tau+\left|\zeta^{\prime}\right|^{2}}-\sqrt{k} \sqrt{\tau+k\left|\zeta^{\prime}\right|^{2}}}{\sqrt{\tau+\left|\zeta^{\prime}\right|^{2}}\left(\sqrt{\tau+\left|\zeta^{\prime}\right|^{2}}+\sqrt{k} \sqrt{\tau+k\left|\zeta^{\prime}\right|^{2}}\right)} \\
& \cdot \exp \left[(t-s) \tau-\left(\xi_{3}+\eta_{3}\right) \sqrt{\tau+\left|\zeta^{\prime}\right|^{2}}\right] .
\end{aligned}
$$


Because of the square root, $g(\tau)$ is a multi-valued function and its branch points are $-\left|\zeta^{\prime}\right|^{2}$ and $-k\left|\zeta^{\prime}\right|^{2}$. A convenient choice for the branch cut is the line segment lying along the real axis running between $\tau=-k\left|\zeta^{\prime}\right|^{2}$ and $\tau=-\left|\zeta^{\prime}\right|^{2}$.

Now we shall convert the line integral running from $\sigma-i \infty$ to $\sigma+i \infty$ into a closed contour so that we can apply the residue theorem. Figure A.2 shows the contour which we will use. The contribution from the $\operatorname{arcs} A B C$ and $J K L$ are negligibly small by Jordan's lemma. The contribution from the line segment $C D$ cancels the contribution from $I J$. Consequently the dumbbell-shaped contour integral shown in Figure A.3 is equivalent to the contour ABCDE FGH I J KL shown in Figure A.2. Because there are no singularities inside the closed contour, the value given by the contour integral shown in Figure A.3 must equal the negative of the integral from $\sigma-i \infty$ to $\sigma+i \infty$.

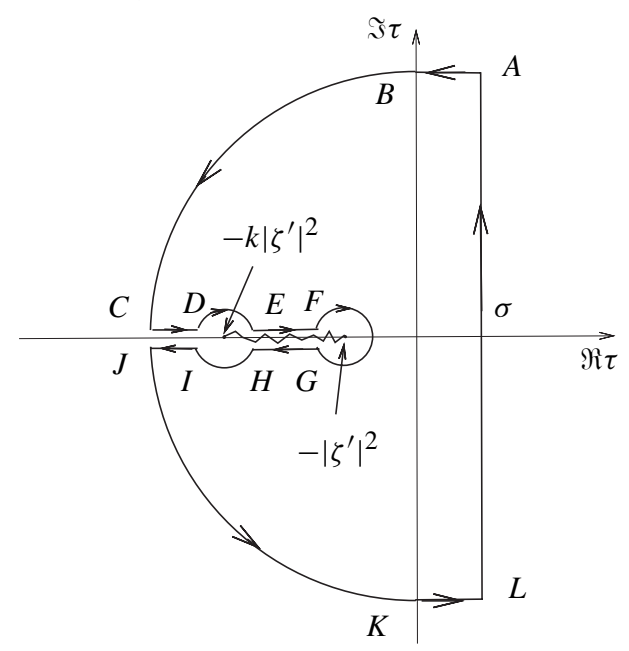

Figure A.2. Contour.

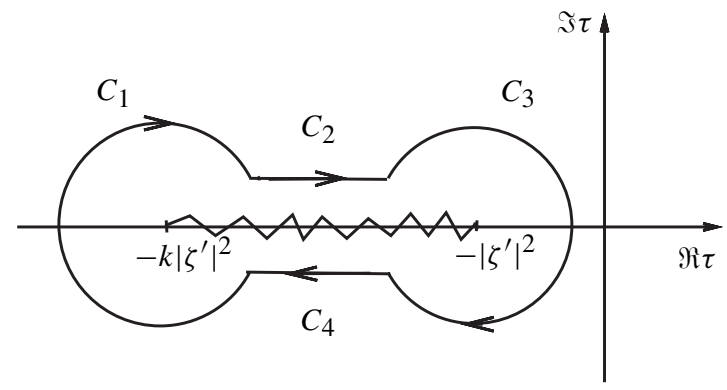

Figure A.3. Dumbbell-shaped contour. 
Along $C_{1}, \tau+k\left|\zeta^{\prime}\right|^{2}=\varepsilon e^{i \theta}$, and $d \tau=i \varepsilon e^{i \theta} d \theta$ so that

$$
\begin{aligned}
& \int_{C_{1}} g(\tau) \mathrm{d} \tau \\
& =\lim _{\varepsilon \rightarrow 0} \int_{2 \pi}^{0} \frac{\sqrt{-k\left|\zeta^{\prime}\right|^{2}+\varepsilon e^{i \theta}+\left|\zeta^{\prime}\right|^{2}}-\sqrt{k} \sqrt{\varepsilon} e^{i \theta / 2}}{\sqrt{-k\left|\zeta^{\prime}\right|^{2}+\varepsilon e^{i \theta}+\left|\zeta^{\prime}\right|^{2}}\left(\sqrt{-k\left|\zeta^{\prime}\right|^{2}+\varepsilon e^{i \theta}+\left|\zeta^{\prime}\right|^{2}}+\sqrt{k} \sqrt{\varepsilon} e^{i \theta / 2}\right)} \\
& \quad \times \exp \left[(t-s)\left(-k\left|\zeta^{\prime}\right|^{2}+\varepsilon e^{i \theta}\right)-\left(\xi_{3}+\eta_{3}\right) \sqrt{-k\left|\zeta^{\prime}\right|^{2}+\varepsilon e^{i \theta}+\left|\zeta^{\prime}\right|^{2}}\right] i \varepsilon e^{i \theta} \mathrm{d} \theta \\
& =\lim _{\varepsilon \rightarrow 0} \int_{2 \pi}^{0} \frac{i \varepsilon e^{i \theta}\left(\sqrt{\varepsilon e^{i \theta}+(k-1)\left|\zeta^{\prime}\right|^{2} e^{\pi i}}-\sqrt{k} \sqrt{\varepsilon} e^{i \theta / 2}\right)}{\sqrt{\varepsilon e^{i \theta}+(k-1)\left|\zeta^{\prime}\right|^{2} e^{\pi i}}\left(\sqrt{\varepsilon e^{i \theta}+(k-1)\left|\zeta^{\prime}\right|^{2} e^{\pi i}}+\sqrt{k} \sqrt{\varepsilon} e^{i \theta / 2}\right)} \\
& \quad \times \exp \left[-(t-s)\left(k\left|\zeta^{\prime}\right|^{2}-\varepsilon e^{i \theta}\right)-\left(\xi_{3}+\eta_{3}\right) \sqrt{\varepsilon e^{i \theta}+(k-1)\left|\zeta^{\prime}\right|^{2} e^{\pi i}}\right] \mathrm{d} \theta \\
& =0 .
\end{aligned}
$$

Along $C_{3}, \tau+\left|\zeta^{\prime}\right|^{2}=\varepsilon e^{i \theta}$ and $d \tau=i \varepsilon e^{i \theta} d \theta$ so that

$$
\begin{aligned}
\int_{C_{3}} g(\tau) \mathrm{d} \tau= & \lim _{\varepsilon \rightarrow 0} \int_{\pi}^{-\pi} \frac{\sqrt{\varepsilon} e^{i \theta / 2}-\sqrt{k} \sqrt{\varepsilon e^{i \theta}-\left|\zeta^{\prime}\right|+k\left|\zeta^{\prime}\right|^{2}}}{\sqrt{\varepsilon} e^{i \theta / 2}\left(\sqrt{\varepsilon} e^{i \theta / 2}+\sqrt{k} \sqrt{\varepsilon e^{i \theta}-\left|\zeta^{\prime}\right|+k\left|\zeta^{\prime}\right|^{2}}\right)} \\
& \times \exp \left[(t-s)\left(-\left|\zeta^{\prime}\right|^{2}+\varepsilon e^{i \theta}\right)-\left(\xi_{3}+\eta_{3}\right) \sqrt{\varepsilon} e^{i \theta / 2}\right] i \varepsilon e^{i \theta} \mathrm{d} \theta \\
= & \lim _{\varepsilon \rightarrow 0} \int_{\pi}^{-\pi} \frac{i \sqrt{\varepsilon} e^{i \theta / 2}\left(\sqrt{\varepsilon} e^{i \theta / 2}-\sqrt{k} \sqrt{\varepsilon e^{i \theta}+(k-1)\left|\zeta^{\prime}\right|}\right)}{\sqrt{\varepsilon} e^{i \theta / 2}+\sqrt{k} \sqrt{\varepsilon e^{i \theta}+(k-1)\left|\zeta^{\prime}\right|}} \\
& \times \exp \left[-(t-s)\left(\left|\zeta^{\prime}\right|^{2}-\varepsilon e^{i \theta}\right)-\left(\xi_{3}+\eta_{3}\right) \sqrt{\varepsilon} e^{i \theta / 2}\right] \mathrm{d} \theta=0 .
\end{aligned}
$$

Along $C_{2}, \tau+k\left|\zeta^{\prime}\right|^{2}=r e^{0 i}, \tau+\left|\zeta^{\prime}\right|^{2}=\left((k-1)\left|\zeta^{\prime}\right|^{2}-r\right) e^{\pi i}$ and $d \tau=d r$ so that

$$
\begin{aligned}
& \int_{C_{2}} g(\tau) \mathrm{d} \tau \\
& =\int_{0}^{(k-1)\left|\zeta^{\prime}\right|^{2}} \frac{\sqrt{(k-1)|\zeta|^{2}-r} e^{\pi i / 2}-\sqrt{k} \sqrt{r}}{\sqrt{(k-1)|\zeta|^{2}-r} e^{\pi i / 2}\left(\sqrt{(k-1)|\zeta|^{2}-r} e^{\pi i / 2}+\sqrt{k} \sqrt{r}\right)} \\
& \quad \times \exp \left[(t-s)\left(-k\left|\zeta^{\prime}\right|^{2}+r\right)-\left(\xi_{3}+\eta_{3}\right) \sqrt{(k-1)|\zeta|^{2}-r} e^{\pi i / 2}\right] \mathrm{d} r \\
& =-i \int_{0}^{1} \frac{\sqrt{k-1}\left|\zeta^{\prime}\right|(\sqrt{r}+i \sqrt{k} \sqrt{1-r})}{\sqrt{r}(\sqrt{r}-i \sqrt{k} \sqrt{1-r})} \\
& \quad \times \exp \left[-(t-s)\left|\zeta^{\prime}\right|^{2}(1+(k-1) r)-i\left(\xi_{3}+\eta_{3}\right) \sqrt{(k-1) r}\left|\zeta^{\prime}\right|\right] \mathrm{d} r .
\end{aligned}
$$


Along $C_{4}, \tau+k\left|\zeta^{\prime}\right|^{2}=r e^{0 i}, \tau+\left|\zeta^{\prime}\right|^{2}=\left((k-1)\left|\zeta^{\prime}\right|^{2}-r\right) e^{-\pi i}, d \tau=d r$ and

$$
\begin{aligned}
& \int_{C_{4}} g(\tau) \mathrm{d} \tau \\
& =\int_{(k-1)\left|\zeta^{\prime}\right|^{2}}^{0} \frac{\sqrt{(k-1)\left|\zeta^{\prime}\right|^{2}-r} e^{-\pi i / 2}-\sqrt{k} \sqrt{r}}{\sqrt{(k-1)\left|\zeta^{\prime}\right|^{2}-r} e^{-\pi i / 2}\left(\sqrt{(k-1)\left|\zeta^{\prime}\right|^{2}-r} e^{-\pi i / 2}+\sqrt{k} \sqrt{r}\right)} \\
& \quad \times \exp \left[(t-s)\left(-k\left|\zeta^{\prime}\right|^{2}+r\right)-\left(\xi_{3}+\eta_{3}\right) \sqrt{(k-1)\left|\zeta^{\prime}\right|^{2}-r} e^{-\pi i / 2}\right] \mathrm{d} r \\
& =-i \int_{0}^{1} \frac{\sqrt{k-1}\left|\zeta^{\prime}\right|(\sqrt{r}-i \sqrt{k} \sqrt{1-r})}{\sqrt{r}(\sqrt{r}+i \sqrt{k} \sqrt{1-r})} \\
& \quad \times \exp \left[-(t-s)\left|\zeta^{\prime}\right|^{2}(1+(k-1) r)+i\left(\xi_{3}+\eta_{3}\right) \sqrt{(k-1) r}\left|\zeta^{\prime}\right|\right] \mathrm{d} r .
\end{aligned}
$$

Thus, we have

$$
\begin{aligned}
h\left(\zeta^{\prime}, t\right)= & \frac{\sqrt{k-1}}{4 \pi} \int_{0}^{1} \frac{\left|\zeta^{\prime}\right|}{\sqrt{r}} \exp \left[-(t-s)\left|\zeta^{\prime}\right|^{2}(k r-r+1)\right] \\
\times & {\left[\frac{\sqrt{r}-i \sqrt{k(1-r)}}{\sqrt{r}+i \sqrt{k(1-r)}} \exp \left\{i\left(\xi_{3}+\eta_{3}\right) \sqrt{(k-1) r}\left|\zeta^{\prime}\right|\right\}\right.} \\
& \left.+\frac{\sqrt{r}+i \sqrt{k(1-r)}}{\sqrt{r}-i \sqrt{k(1-r)}} \exp \left\{-i\left(\xi_{3}+\eta_{3}\right) \sqrt{(k-1) r}\left|\zeta^{\prime}\right|\right\}\right] \mathrm{d} r
\end{aligned}
$$

\section{References}

[1] G. AlesSANDrini and M. Di CRISTO, Stable determination of an inclusion by boundary measurements, SIAM J. Math. Anal. 37 (2005), 200-217.

[2] D. G. ARonson, Non-negative solutions of linear parabolic equations, Ann. Scuola Norm. Sup. Pisa, Cl. Sci. 22 (1968), 607-694.

[3] W. J. CANTWELL and J. Morton, The significance of damage and defects and their detection in composite materials: A review, J. Strain Anal. Eng. Des. 27 (1992), 29-42.

[4] Y. DAIDO, H. KANG and G. NAKAMURA, A probe method for the inverse boundary value problem of non-stationary heat equations, Inverse Problems 23 (2007), 1787-1800.

[5] M. Di Cristo, K. Kim and G. NAKAMURA, Estimate of the fundamental solution for parabolic operators with discontinuous coefficients, preprint (http://arxiv.org).

[6] M. Di Cristo and S. Vessella, Stable determination of the discontinuous conductivity coefficient of a parabolic equation, preprint (http://arxiv.org).

[7] A. ELAYYAN and V. ISAKOV, On uniqueness of recovery of the discontinuous conductivity coefficient of a parabolic equation, SIAM J. Math. Anal. 28 (1997), 49-59.

[8] A. Friedman, "Partial Differential Equations of Parabolic Type", Prentice-Hall, INC. 1964.

[9] P. GReINER, An asymptotic expansion for the heat equation, Arch. Ration. Mech. Anal. 41 (1971), 163-218.

[10] L. Hörmander, "The Analysis of Linear Partial Differential Operator III", A series of comprehensive study in Mathematics, Springer-Verlag, 1985. 
[11] M. IKEHATA, Reconstruction of inclusion from boundary measurements, J. Inverse IllPosed Probl. 10 (2002) 37-65.

[12] V. Isakov, "Inverse Problems for Partial Differential Equations", Applied Mathematical Sciences, Vol. 127, Springer 1988.

[13] V. D. KupradzE, "Three-dimensional Problems of the Mathematical Theory of Elasticity and Thermoelasticity", North-Holland series in Applied Mathematics and Mechanics, Vol. 25, North-Holland Publishing Company, 1979.

[14] G. M. Lieberman, "Second Order Parabolic Differential Equations", World Scientific Publishing Co., Inc., 1996.

[15] Y. Y. Li and L. Nirenberg, Estimates for elliptic systems from composite material, Comm. Pure Appl. Math., 56 (2003), 892-925.

[16] P. M. PATEL, S. K. LAU and D. P. ALMOND, A review of image analysis techniques applied in transient thermographic nondestructive testing, Nondestruct. Test. Eval. 6 (1992) 343364.

[17] P. Reulet, D. Nortershauser and P. Millan, Inverse method using infrared thermography for surface temperature and heat flux measurements, 20th International Congress on Instrumentation in Aerospace Simulation Facilities, 2003, ICIASF '03, 118-126.

[18] L. YI, K. KIM and G. NAKAMURA, Numerical implementation of dynamical probe method, J. Comput. Math. 28 (2010), 87-104.

[19] J. WloKA, "Partial Differential Equations", Cambridge University Press, 1987.

Department of Mathematics and Statistics

Wichita State University

Wichita, 67260-0033, USA

victor.isakov@wichita.edu

Department of Mathematics

Hokkaido University

Sapporo 060-0810, Japan

kim@math.sci.hokudai.ac.jp

gnaka@math.sci.hokudai.ac.jp 